\title{
A gradient border model for cell fate decisions at the neural plate border
}

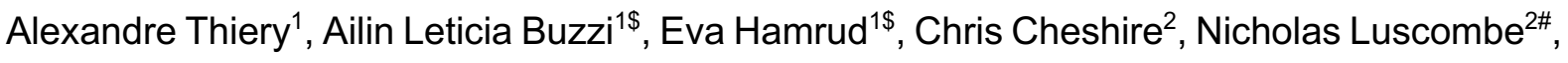 \\ James Briscoe ${ }^{2}$, Andrea Streit ${ }^{1 *}$ \\ 1 Centre for Craniofacial and Regenerative Biology, Faculty of Dentistry, Oral and Craniofacial Sciences, King's \\ College London, London, UK. \\ 2 Bioinformatics and Computational Biology Laboratory, The Francis Crick Institute, 1 Midland Road, London, UK. \\ \# Current address: Genomics and Regulatory Systems Unit, Okinawa Institute of Science and Technology \\ Graduate University, Japan.
}

\$These authors contributed equally to this work.
${ }^{*}$ Corresponding author: andrea.streit@kcl.ac.uk

\section{Abstract}

The vertebrate 'neural plate border' is a transient territory at the edge of the neural plate containing precursors for all ectodermal derivatives: the neural plate; neural crest; placodes; and epidermis. Elegant functional experiments in a range of vertebrate models have provided an in-depth understanding of gene regulatory interactions within the ectoderm. However, these experiments conducted at tissue level have raised seemingly contradictory models for fate allocation of individual cells. Here, we carry out single cell RNA sequencing of chick ectoderm from primitive streak to neurulation stage, to explore cell state diversity and heterogeneity. We identify and characterise the dynamics of gene modules containing key factors known to regulate ectodermal cell fates, allowing us to model the order in which these fates are specified. Furthermore, we find that genes previously classified as neural plate border specifiers typically exhibit dynamic expression patterns and are biased towards either placodal or neural crest fates. Instead, given its transient and heterogenous characteristics, the neural plate border should be defined based on the co-expression of alternative placodal and neural crest gene modules. Finally, we propose a 'gradient border' model for cell fate choice at the neural plate border, with the probability of cell fate allocation closely tied to the spatiotemporal positioning of cells. 


\section{Introduction}

2

The vertebrate nervous system is arguably the most complex organ of our body. During development, it arises from only three progenitor populations: the neural plate generates the entire central nervous system, while neural crest and sensory placodes jointly form the peripheral nervous system. Placode derivatives are confined to the head contributing to sense organs and cranial ganglia, while neural crest cells form along the entire body axis to generate various cell types including neurons and glial as well as parts of the craniofacial skeleton, cartilage and teeth (for review: Baker \& Bronner-Fraser, 1997; Baker \& Bronner-Fraser, 2001; Grocott et al., 2012; Patthey et al., 2014; Pla \& Monsoro-Burq, 2018; Prasad et al., 2019; Simoes-Costa \& Bronner, 2015).

The neural plate becomes morphologically distinct shortly after gastrulation. However, neural, neural crest and placode precursors continue to be intermingled at its edge, in a territory termed the neural plate border (NPB) that is characterised by the co-expression of neural and non-neural markers as well as so-called NPB specifiers (Pla \& Monsoro-Burq, 2018; Roellig et al., 2017; Streit, 2002; Thiery et al., 2020). As the neural plate invaginates, placode precursors remain in the surface ectoderm, while neural crest cells are largely incorporated into neural folds from where they delaminate and migrate to their final destination. Although the induction of these three fates has been studied for decades, some of the most fundamental questions remain unresolved. For example, we do not know when and how these lineages segregate, whether they do so in a specific order and how individual cells make these decisions.

Modulation of different signalling pathways plays a crucial role in controlling the fate of all ectodermal cells. FGF signalling is required for the specification of neural, neural crest and placode precursors and for positioning the NPB (Ahrens \& Schlosser, 2005; Litsiou et al., 2005; Londin et al., 2005; Monsoro-Burq et al., 2003; Streit et al., 2000; Streit \& Stern, 1999; Stuhlmiller \& García-Castro, 2012; Wilson et al., 2000; Yardley \& Garcia-Castro, 2012). While BMP signalling must be inhibited for neural development (Hemmati-Brivanlou et al., 1994; Linker \& Stern, 2004; Londin et al., 2005; Sasai et al., 1995), it is necessary for placode precursor formation at gastrulation, but must be switched off thereafter (Ahrens \& Schlosser, 2005; Kwon et al., 2010; Litsiou et al., 2005). Likewise, neural crest cells need BMP activity early, but unlike placodes continue to do so as they are specified (Barth et al., 1999; Kwon et al., 2010; Liem et al., 1995; Marchant et al., 1998; Steventon et al., 2009). Finally, Wnt activity is required for neural crest cell induction (Garcia-Castro et al., 2002; Steventon et al., 2009) but must be inhibited for neural and placode precursors to form (Heeg-Truesdell \& LaBonne, 
2006; Litsiou et al., 2005; Patthey et al., 2008). These signals activate different transcription factor networks in a temporally and spatially controlled manner that in turn impart cell identity and have therefore been termed fate 'specifiers'. For example, Msx1 and Pax3 have been implicated as NPB specifiers (Milet et al., 2013; Monsoro-Burq, 2015; Plouhinec et al., 2014), while Pax7 (Basch et al., 2006) and Six1 (Brugmann et al., 2004; Chen et al., 2009; Christophorou et al., 2009; Ozaki et al., 2004) are involved in neural crest and placode precursor specification, respectively. These factors appear to act in feed forward loops, upregulating their own expression while simultaneously repressing factors that specify alternative fates (for review: Grocott et al., 2012; Thiery et al., 2020).

Based on these studies combined with tissue transplantation experiments, two apparently contradictory models for the segregation of neural, neural crest and placodal fates have emerged. The 'binary competence model' suggests that an initial step in lineage segregation entails a subdivision of the ectoderm into neural/neural crest and placode/epidermal territories (Pieper et al., 2012; Schlosser, 2014). This implies a closer relationship of neural crest cells with the central nervous system and of placodes with the epidermis. In contrast, the 'NPB model' suggests that cells at the border of the neural plate have mixed identity and retain the ability to generate all ectodermal derivatives until after neurulation begins (Roellig et al., 2017; Schlosser, 2014; Thiery et al., 2020). Studies in Xenopus have taken this idea a step further, revealing that unlike other ectodermal cells, neural crest or NPB cells retain pluripotency markers and that this is key for their ability to contribute to multiple fates (Buitrago-Delgado et al., 2015; Prasad et al., 2012). In support of this idea, the NPB region is initially characterised by co-expression of neural and non-neural markers (for review: Grocott et al., 2012; Pla \& Monsoro-Burq, 2018; Thiery et al., 2020). Fate specifier genes are activated within this territory in partially overlapping patterns to resolve into distinct domains as neurulation begins. While both models are attractive and provide working hypotheses, the underlying experiments were analysed at cell population, rather than at single cell level. They therefore do not address how individual NBP cells make decisions, nor whether they are multipotent or a population of mixed progenitors that are already biased towards their later identity. Recent findings have proposed that prior to fate commitment cells show increasing levels of heterogeneity in gene expression and do not exhibit a definitive transcriptional signature (Soldatov et al., 2019). It is therefore possible that NBP cells represent such undetermined progenitor cells.

Recent studies in chick have begun to explore these questions. Single cell analysis at the NPB reveals considerable heterogeneity of gene expression: factors previously thought to be fatespecific are co-expressed in individual cells even as late as neural tube stages (Roellig et al., 2017; Williams et al., 2022). Furthermore, lineage tracing using a 'neural-specific' Sox2 
enhancer shows that Sox $2^{+}$cells can contribute not only to neural tissue but also to neural crest and epidermis (Roellig et al., 2017). Together, these findings suggest that cell fate allocation at the NPB occurs over a much longer developmental period than previously thought and that NPB cells retain plasticity until early neurulation. However, although some factors have been identified, the underlying molecular mechanisms of how individual cells make decisions at the NBP remain poorly understood.

Here, we explored the transcriptional changes as cells transit from the neural plate border at early primitive streak to definitive neural, neural crest and placodal fates at single cell level. In total we present single cell RNAseq (scRNA) data for 17,992 high quality cells. Rather than using 2-3 molecular markers to assign cell identity, we established a binary knowledge matrix using previously published in situ hybridisation expression data. This allows us to classify cells at each developmental stage, despite the high levels of heterogeneity observed. We find that prior to head fold stages cells do not fall into transcriptional distinct groups but are characterised by graded and partially overlapping gene expression. Their organisation largely reflects their position along the anterior-posterior and medial-lateral axis in the embryo. As expected, cell diversity increases as development proceeds, with a division of neural and nonneural fates becoming apparent at the first somite stage together with the emergence of neural crest cells. By the 8-somite stage, neural, neural crest and placodal cells are largely segregated, although even at this late stage we find cells with heterogenous expression profiles.

Our analysis reveals that NPB cells can already be identified at primitive streak stages by the overlapping expression of neural and non-neural genes, suggesting that this cell state is established before NPB specifiers are activated. Using a gene module approach, we identify groups of genes with dynamic changes in our dataset that are characteristic for neural, neural crest and placodal fates. Comparing co-expression of whole gene modules (rather than individual transcripts) reveals that NPB cells co-express gene modules which later become restricted to placodal or neural crest states. This suggests that NPB cells are in an undecided transcriptional state and may have the potential to give rise to both (or more) fates. Interestingly, although we did not find NPB cells as defined by classical NPB markers at ss4, we did identify cells that co-express competing placodal and neural crest programmes. These cells connect segregating cell populations, resemblant of previously characterised bridge cells in the differentiating murine neural crest which exhibit high heterogeneity prior to segregation (Soldatov et al., 2019). Here, we propose that 'bridge cells' between placodal and neural crest populations continue to be plastic, maintaining the ability to contribute to different fates. Overall, our analysis has led us to revisit the definition of the NPB and propose that NPB cells 
may be better defined by the co-expression of competing gene modules rather than a particular gene signature.

\section{$\underline{\text { Results }}$}

\section{Classification of ectodermal cells using a binary knowledge matrix of known marker genes}

Neural, neural crest and placode progenitors arise from the embryonic ectoderm. However, how individual cells diversify and the sequence and timing of these events remain to be elucidated. To capture their segregation, we carried out 10x Genomics single-cell mRNA sequencing (scRNAseq) on cells taken from a broad region of the chick epiblast at six developmental stages: $\mathrm{HH} 4 / 4$ (primitive streak), $\mathrm{HH}$ (head process), $\mathrm{HH} 6$ (head fold), $\mathrm{HH} 7$ (1 somite), somite stage (ss) 4 and ss 8 (Figure 1A). These stages were chosen because they encompass the initial formation of the neural plate to the beginning of neurulation when these fate decisions are thought to take place. After integration across batches, stringent quality control and removal of contaminating cell populations (see methods and Figure 1-figure supplement $1 \mathrm{~A}-\mathrm{I}$ ), we captured a total of 17,992 high quality cells with approximately $2500-$ 4000 cells per stage and an average of 3924 genes per cell. Dimensionality reduction and UMAP embedding showed that cells were organised primarily by stage (Figure 2E). Later stages displayed higher cell diversity as compared to earlier stages where most cells were transcriptionally similar (Figure 1B-E and Figure 2A-D).

Typically, scRNAseq cell states are classified using a small number of definitive marker genes which exhibit mutually exclusive expression patterns. However, this approach is difficult to apply to the NPB for several reasons. Firstly, the early NPB cannot be classified using definitive markers as it emerges in the region of overlap between neural and non-neural genes. Secondly, the NPB is a transitory state through which cells are thought to pass prior to acquisition of any of the four ectodermal fates (neural, neural crest, placodal and epidermal). Finally, cells at the NPB appear to be highly heterogeneous, with precursors for the different ectodermal fates found intermingled, even at late stages of neurulation (Bhattacharyya et al., 2004; Roellig et al., 2017; Streit, 2002; Williams et al., 2022). To overcome these challenges, we categorised cells using a binary knowledge matrix based on expression data obtained from the literature (Supplementary File 1). We examined available in situ hybridisation data of 76 genes with known regionalised expression patterns and binarized their expression within each 
defined cell state. This allowed us to define a total of 24 cell states spanning primitive streak to neural tube stages.

151 We first unbiasedly clustered cells from each developmental stage at a high resolution to ensure capturing the full diversity of cell states (Figure 1-figure supplement $1 \mathrm{~J}$ ). Clusters were then classified based on the similarity between their gene expression and the knowledge matrix (see methods). Next, cells from all stages were clustered together and cell state labels transferred to the entire data set (Figure 2F). This classification approach successfully identified 22 distinct cell states within the ectoderm. As expected, cell diversity increases as development proceeds. Early NPB and early neural cell states can already be identified at

158 primitive streak stages, while placode progenitor and neural crest states emerge at head process and ss4, respectively (Figure 1B and Figure 2A-B). 

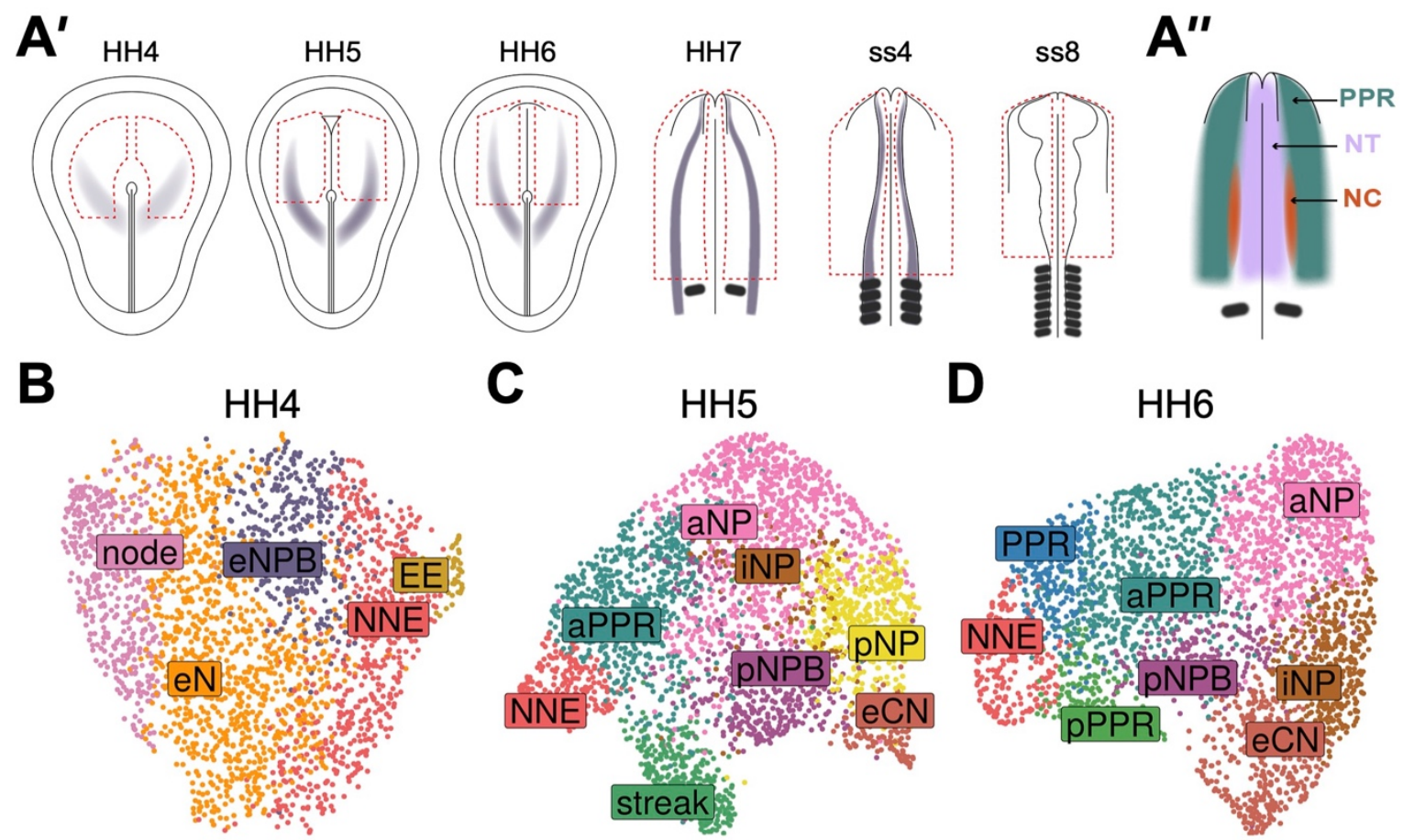

D $\mathrm{HH} 6$
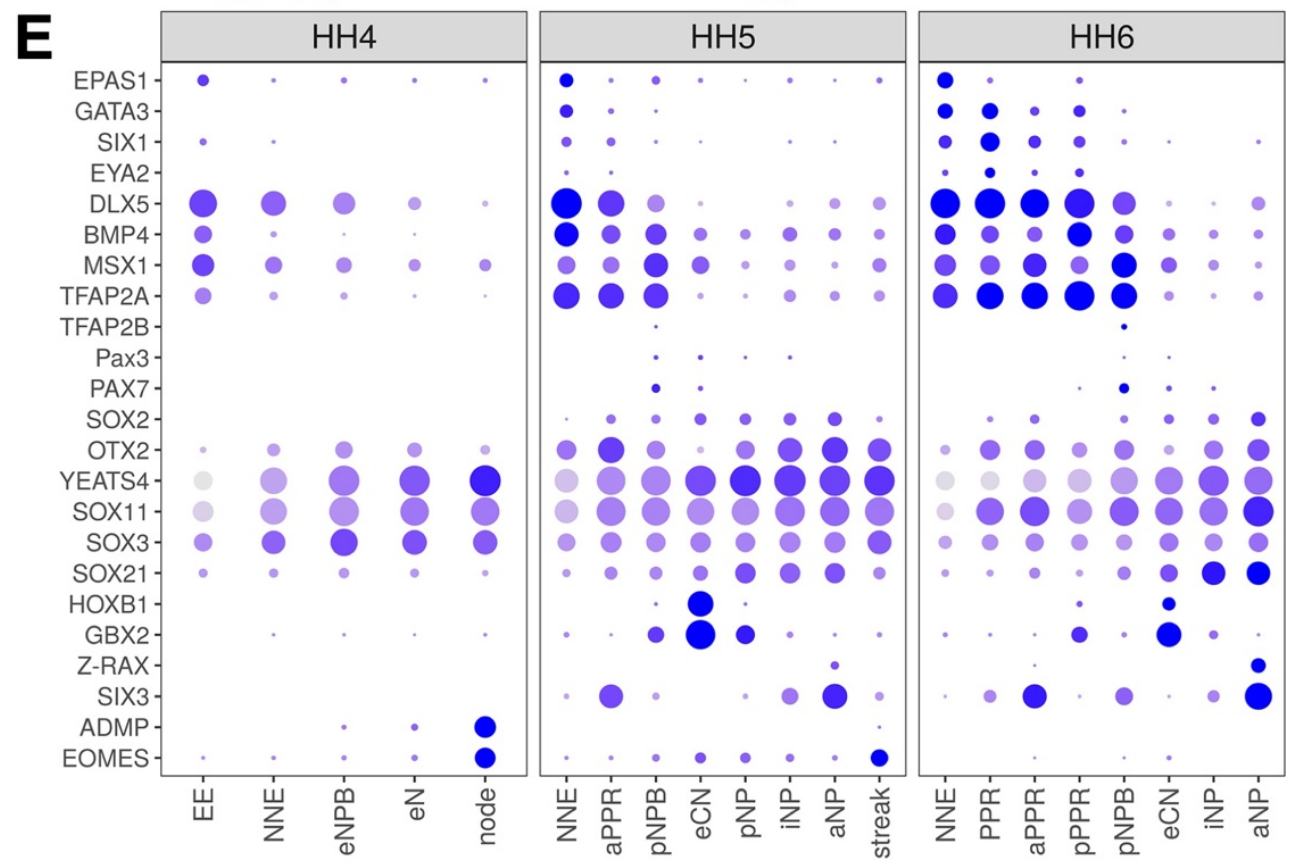

F $D 1 \times 5$

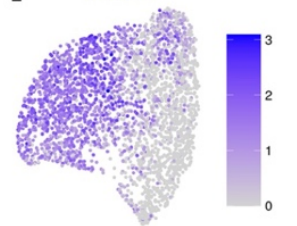

G Six1

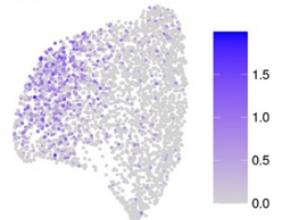

H $\operatorname{Pax} 7$

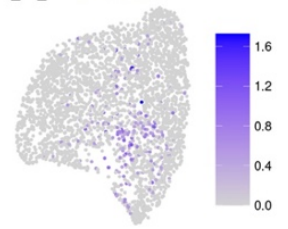

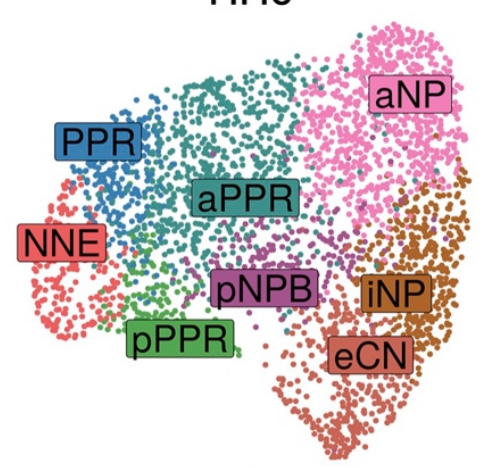

pPPR
Average

expression

1.0

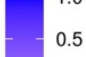

$-0.0$

$-0.5$

$-1.0$

Percent cells expressing

- 25

- 50

- 75
I Sox21

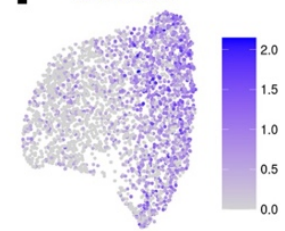

J Yeats4

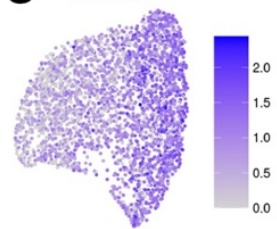

Figure 1. Cells at stages $\mathrm{HH} 4-\mathrm{HH} 6$ reflect the anterior-posterior and medio-lateral axes in the embyro. (A') Dorsal view schematics of chick embryo at stages HH4-ss8 depicting the ectodermal tissue region dissected for 10x scRNAseq (red dotted line). Purple shading illustrates previously characterized region of Pax7 expression (Basch et al. 2006). (A") Schematic of a 1-somite stage $(\mathrm{HH} 7)$ chick embryo illustrating the pre-placodal region (PPR), 
neural crest (neural crest) and neural tube (NT). (B-D) UMAP plots for cells collected at $\mathrm{HH}^{-}$ 14 (primitive streak), $\mathrm{HH} 5$ (head process), $\mathrm{HH} 6$ (head fold) stages. Cell clusters are coloured and labelled based on their semi-unbiased cell state classifications (binary knowledge matrix available in Supplementary File 1). (E) Dot plots displaying the average expression of key marker genes across cell states at $\mathrm{HH} 4, \mathrm{HH} 5$ and $\mathrm{HH} 6$. The size of the dots represents the number of cells expressing a given gene within each cell population. (F) Feature plots revealing the mediolateral segregation of key marker genes at $\mathrm{HH} 6$.

\section{Cell states emerging from primitive streak to head fold stages}

To explore the changes in cell diversity within the embryonic ectoderm over time, we compared cell states present at different stages and identified the time point when each emerged. We visualised cell transcriptomes in low dimensionality space using UMAPs, in turn allowing us to infer relationships between cell states and explore the distribution of gene expression across the dataset. We first examined stages $\mathrm{HH} 4-\mathrm{HH} 6$ to characterise events as the neural plate is established and placodal and neural crest cells are thought to be specified.

An early NPB state is identified at primitive streak stages prior to the expression of NPB specifiers

By primitive streak stage, mediolateral (M-L) gradients of molecular markers demarcate preneural and non-neural territories within the chick epiblast (Pera et al., 1999; Rex et al., 1997; Sheng \& Stern, 1999; Streit et al., 2000; Streit et al., 1998). At HH4, unbiased clustering clearly organises cells in a pattern reminiscent of this $\mathrm{M}-\mathrm{L}$ axis (Figure $1 \mathrm{~B}$; from left to right) as evidenced by the expression of node (ADMP), pre-neural (Yeats4, Sox3, Sox11) and nonneural (DIx5, Bmp4, Msx1, Tfap2a) genes (Figure 1B, E and Figure 1-figure supplement 2A). Cell states can be broadly characterised based on combinatorial gene expression (Figure 1E) and we identify five cell states: node, early neural (eN), early neural plate border (eNPB), nonneural ectoderm (NNE) and extraembryonic ectoderm (EE) (Figure 1B). While some genes are shared between many cells, early neural plate cells are defined by high levels of pre-neural transcripts while the non-neural and extraembryonic ectoderm are characterised by increased non-neural gene expression. Notably, we find that an early NPB state can be identified by the co-expression of both neural and non-neural genes. Thus, NPB cells are already apparent prior to the differential upregulation of genes previously characterised as NPB specifiers (Msx1, Pax7, Tfap2a, Bmp4, DIx5, Pax3) (Basch et al., 2006; Ezin et al., 2009; Hong \& SaintJeannet, 2007; McLarren et al., 2003; Rothstein \& Simoes-Costa, 2020; Streit \& Stern, 1999) (for review: Simoes-Costa \& Bronner, 2015; Thiery et al., 2020).

The first emerging NPB and PPR cells have regional identity 
At $\mathrm{HH} 5$, cell positions in the UMAP continue to reflect organisation along the M-L axis in the embryo (Figure 1C), but new transcriptional states emerge. Whilst non-neural ectoderm cells display a similar expression profile at $\mathrm{HH} 5$ compared to $\mathrm{HH} 4$, neural plate cells differ from early neural cells in their expression of definitive neural (e.g., Sox2) and regional markers (Figure 1E; Figure 1-figure supplement 2B). Like at $\mathrm{HH} 4$, many cells continue to co-express neural and non-neural transcripts (Figure 1-figure supplement 2B). Within the latter cell group, we observe the upregulation of PPR genes (Six1, Eya2; Figure 1C, E: aPPR, anterior PPR) and for the first time NPB specifiers (Msx1, Pax3, Pax7; Figure 1C, E: pNPB, posterior NPB). These observations correlate well with gene expression in the embryo. Msx1 is initially expressed in the extraembryonic and non-neural ectoderm, and together with Pax 7 begins to be upregulated in the NPB thereafter (Basch et al., 2006; Streit \& Stern, 1999), while PPR markers are first expressed at HH5. Notably, the first NPB cells to emerge have posterior character, while placode precursors have anterior identity based on their expression of Gbx2 and Six3, respectively (Figure 1E; see also below).

\section{Anteroposterior organisation of cells at head process and head fold stages}

Cells with distinct anteroposterior (A-P) character are first observed at HH5 through the restricted expression of various homeobox transcription factors. Rax and HoxB1 are highly confined to distinct cell groups, whilst in contrast Six3 and Gbx2 exhibit broader negatively correlated expression gradients across cell clusters with some degree of overlap (Figure 1E and Figure 1-figure supplement $2 \mathrm{~B}$ ). The organisation of cells within the UMAP reflects the A$P$ expression gradients of these genes in the embryo (Chapman et al., 2002; Hidalgo-Sanchez et al., 2005; Ohuchi et al., 1999; Paxton et al., 2010). The overlap of A-P factors was used to distinguish different NP, PPR and NPB regions during unbiased cell state classification (Supplementary File 1).

Within the NP we can distinguish intermediate neural plate (iNP) states from the anterior and posterior neural plate (aNP, pNP) and from early caudal neural $(\mathrm{eCN})$ states. However, within the PPR and NPB we identify only anterior (aPPR; Six $3^{+}$) and posterior (pNPB; Gbx2+) cell states, respectively. While in UMAPs aPPR cells are located between the NNE and anterior NP, the pNBP cluster resides between the iNP and pPPR clusters (Figure 1C, E Figure 1figure supplement $2 \mathrm{C}$ ), reflective of both its $\mathrm{A}-\mathrm{P}$ and $\mathrm{M}-\mathrm{L}$ position in vivo.

Between HH5 and HH6, posterior Gbx2 ${ }^{+}$PPR (pPPR) cell states emerge (Figure 1D), as do PPR cells that appear to lack regional identity. At this stage, Otx2 and Gbx2 expression domains in the embryo abut (Steventon et al., 2012), suggesting that cells with generic PPR character might be intermingled with those already biased towards axial identity and either 
retain the potential to contribute to multiple axial levels or will later become intermediate PPR cells.

Together, our data show that the emergence of cells with divergent transcriptional profiles resembles patterning of the ectoderm in vivo. However, unbiased cell clustering forces cells into discrete clusters which may not fully reflect heterogeneity in gene expression and cell diversity. Indeed, our single cell data reveal the heterogenous nature of gene expression: markers are broadly expressed and are not confined by cluster boundaries or by neighbouring expression domains (Figure 1F-J, Figure 1-figure supplement $2 \mathrm{C}$ ). Despite this heterogeneity, our cell classification approach successfully identifies increased ectodermal cell diversity over time, with neural, posterior NPB and anterior PPR cells emerging when the definitive neural plate forms. Importantly, the relative positions of these cell states in the UMAPs reflect their location in the embryo.

\section{Increasing cell diversity from 1-somite to 8-somite stages}

At $\mathrm{HH} 7$, the neural folds begin to elevate indicating the start of neurulation, while during early somitogenesis neural crest cells start to migrate and placode precursors begin to diversify. We therefore sought to characterise the transcriptional changes at single cell level as neural, neural crest and placodal fates emerge.

\section{Emergence of neural crest cells and segregation of neural/non-neural fates}

Following clustering, we identified two major superclusters at $\mathrm{HH} 7$, one containing neural tube and neural crest and the other placodal and future epidermal clusters (Figure 2A). These superclusters appear to be largely separate, both by their UMAP embedding and the restricted expression of definitive fate markers (Figure 2A, D). Whereas at earlier stages many cells coexpress non-neural and neural markers (Figure 1E, F, J), at $\mathrm{HH} 7$ cells expressing nonneural/placodal (D/x5, Six1) and neural transcripts (Sox21, Sox2) are largely confined to their respective supercluster (Figure 2-figure supplement $1 \mathrm{~A}$ ). It is at this stage that anterior NPB cells can first be identified transcriptionally, and together with posterior NPB cells they connect the two superclusters (Figure 2A; aNPB, pNPB).

Adjacent to the NPB clusters, we observe a neural crest cell cluster expressing early definitive neural crest markers including Tfap2b, Snai2 and Axud1 (Csrnp1) alongside NPB markers (Msx1, Pax7 and Tfap2a) (Figure 2D; NC and Figure 2-figure supplement 1A). Thus, neural crest cells first emerge as a distinct cell population at $\mathrm{HH}$. Although many genes are shared between the NPB and neural crest cell clusters, NPB cells also express PPR and non-neural 
markers (Six1, Eya2 and D/x5) which are in turn downregulated in neural crest cells (Figure 2D and Figure 2-figure supplement 1A). Furthermore, we find that Msx1 and Pax7 are never strongly expressed within placodal cell clusters. It is therefore possible that these NPB specifiers may not represent NPB markers, but rather an early bias towards neural crest fate.

'Bridge cells' identified as putative undetermined cells undergoing fate decisions during neurulation

At ss4 and ss8, NPB cells can no longer be identified using the above criteria. Instead, we observe an increasing diversity of cells classified as placodal, neural crest or neural (Figure $2 B, C)$. Within the neural cell population, forebrain, midbrain, and hindbrain ( $F B, M B, H B)$ clusters are clearly distinguishable. Six 3 and Gbx2 expression define cells with fore- and hindbrain identity, respectively, whilst the upregulation of the dorsal midbrain marker Wnt4 (Hollyday et al., 1995) and mid/hindbrain boundary marker Pax2 (Hidalgo-Sanchez et al., 1999) defines the midbrain-like cells (Figure 2D and Figure 2-figure supplement 1B). As development progresses, neural, neural crest and placode cells become increasingly distinct, with neural crest cells beginning to express a suite of definitive neural crest markers, including Sox10 in the delaminating neural crest (dNC) cluster (Figure 2D and Figure 2-figure supplement 1B, C).

Despite the apparent segregation of neural, neural crest and placodal fates during ss 4 and ss8, connecting cells bridge PPR clusters with both neural crest and forebrain clusters, as well as neural crest with midbrain cell clusters in a four-way pattern reflecting their A-P and M-L segregation in vivo (Figure $2 \mathrm{~B}, \mathrm{C}$ ). To predict the directionality of transcriptional change within these bridge cells, and hence their potential developmental trajectory, we performed RNA velocity analysis (Bergen et al., 2020; La Manno et al., 2018). This analysis predicts that bridge cells move away from cluster boundaries, towards definitive cell states (Figure 2G, H). This observation implies that bridge cells do not have a definitive transcriptional signature and may retain plasticity to contribute to different fates, despite the absence of a distinct NPB state from ss4 onwards.

Together our data show that whereas cell states at $\mathrm{HH} 4-\mathrm{HH} 6$ exhibit broad overlapping gene expression, definitive fate markers are upregulated from $\mathrm{HH} 5$ onwards and subsequently gene expression is refined at the boundaries between cell clusters. At $\mathrm{HH} 7$ and ss4, placodal, neural crest and neural fates begin to segregate. However, even as the neural tube closes, bridge cells continue to straddle cluster boundaries; these cells may be transcriptionally indeterminate and therefore still actively undergoing fate decision processes. 

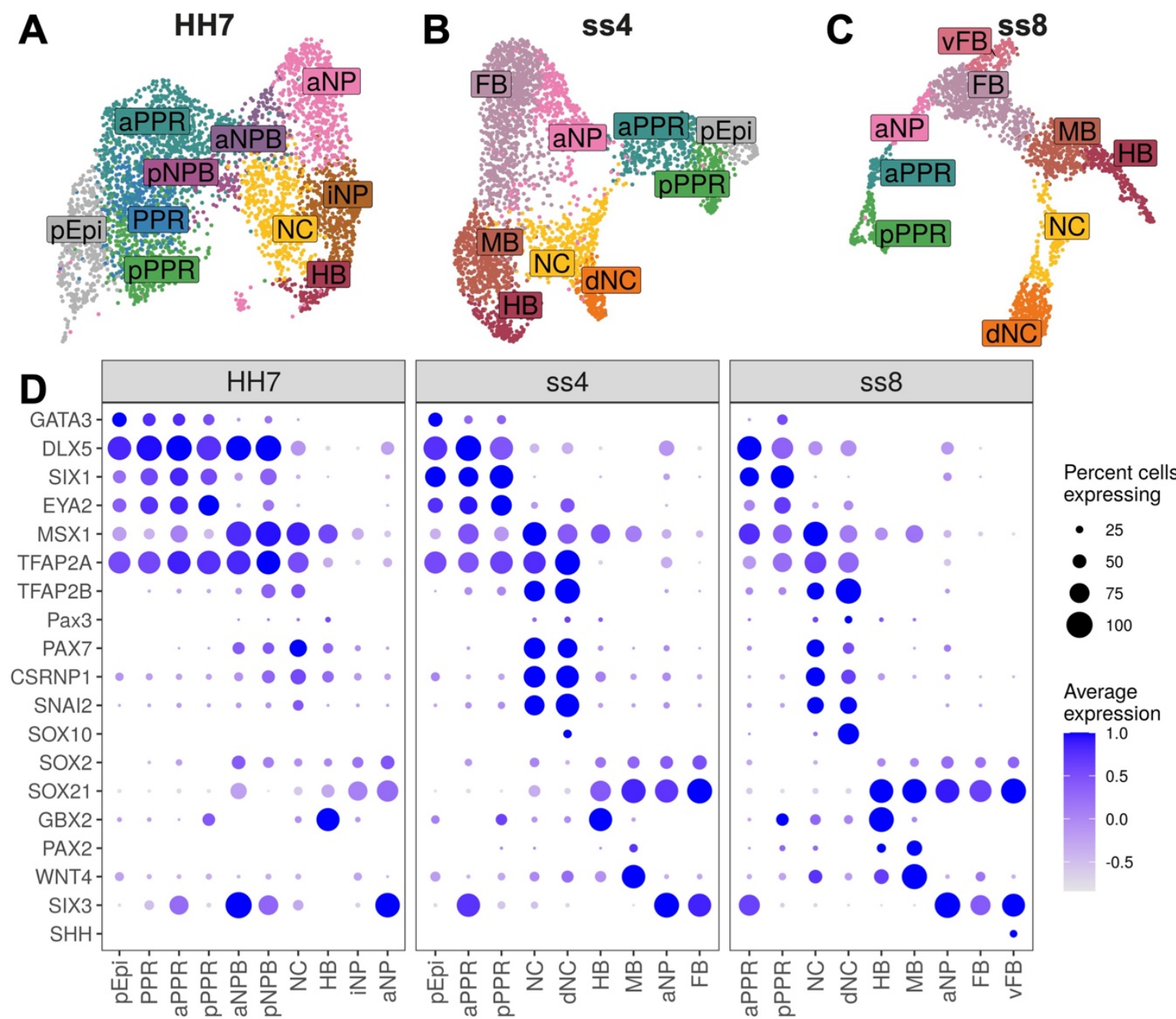

$\mathbf{E}$

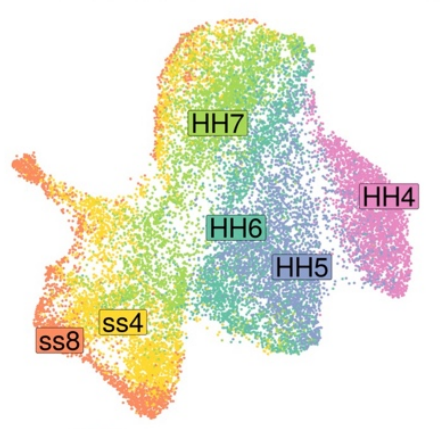

$\mathbf{G}$

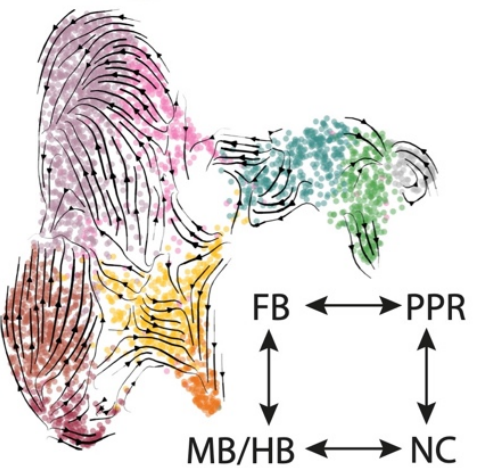

$\mathbf{F}$

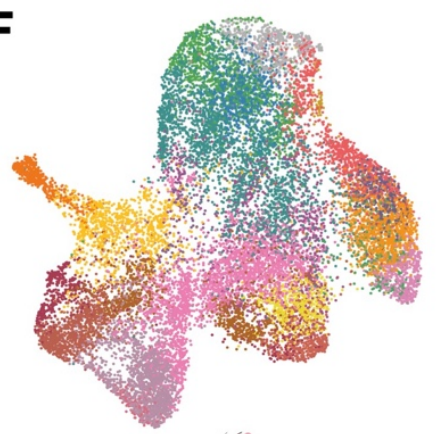

H

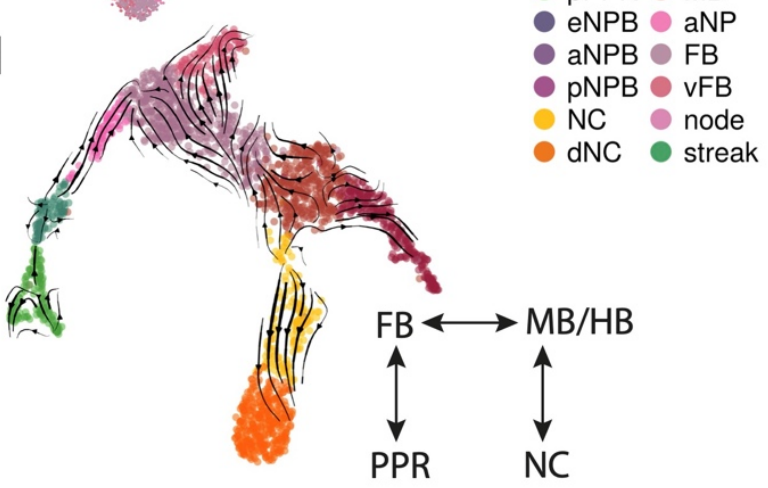

Figure 2. Increased cell diversity and lineage segregation from HH7 to ss8. (A-C) UMAP plots for cells collected at $\mathrm{HH} 7$ (1 somite stage), ss4 (4 somite stage) and ss8 (8 somite stage). Cell clusters are coloured and labelled based on their semi-unbiased cell state classifications 
(binary knowledge matrix available in Supplementary File 1). (D) Dot plots displaying the average expression of key marker genes across cell states at $\mathrm{HH} 7$, ss 4 and ss8. The size of the dots represents the number of cells expressing a given gene within each cell population. (E) UMAP plot for the whole dataset (HH4-ss8 combined) coloured and labelled by stage. (F) UMAP plot for the whole dataset coloured by cell state classifications. Cell state classifications were calculated independently for each stage and transferred across for visualisation of the full combined dataset. (G-H) UMAP plots for ss4 and ss8 overlaid with RNA velocity vectors depicting the predicted directionality of transcriptional change. Cells are coloured by cell state classification (shown in B and $C$ ). Schematics summarise the key fate segregation events taking place at these stages predicted by RNA velocity analysis.

\section{Dynamic changes in gene module expression over time}

Many transcriptional regulators and their interactions have been implicated in cell fate decisions as the ectoderm is specified and differentiates into its derivatives. However, in this context studies have largely taken a gene candidate approach. To explore the full transcriptional dynamics of both known and new transcriptional regulators during the specification of neural, neural crest and placodal cells, we modelled the dynamics of whole gene modules rather than individual candidate genes. This allows us to investigate transcriptional dynamics in an unbiased manner whilst simultaneously minimising the loss of potentially important factors.

\section{Defining neural, neural crest and placodal gene modules}

To investigate the segregation of neural, neural crest and placode fates, we first sought to identify gene modules that characterise each population. Gene modules are groups of genes that display similar expression profiles across a dataset. We calculated gene modules using the Antler R package (Delile et al., 2019), which iteratively clusters genes hierarchically based on their gene-gene Spearman correlation. This approach reveals 40 modules each showing highly correlated gene expression across all stages (HH4-ss8). To focus on gene modules which may include candidates for fate segregation, we included only those that are differentially expressed between any cell state and the rest of the dataset, and then filtered to remove those with differential expression between sequencing batches. Finally, since our data show that the segregation of neural, neural crest and placodal cell states is almost complete at ss8 (Figure 2C), we kept those gene modules that show differential expression between at least one of the three fates at ss8 (see methods). This resulted in nine modules that were considered further (Figure 3-figure supplement 1A and Figure 3-Source Data 1). Of these, four gene modules (GM21-24) are specifically expressed in the PPR at ss8, one (GM13) in 
neural crest cells, and the remaining four $(G M 7,9,11,20)$ are upregulated primarily in neural clusters (Figure 3-figure supplement 1A).

When examining the genes within each module, we noticed that GM20 and GM22 include genes with widespread expression or expression in the lateral mesoderm and/or the extraembryonic region (Epas1, Nkx2-5, Tnnc1, Pgk1) (Adams et al., 2008; Bell et al., 2004; Liberatore et al., 2000; Ota et al., 2007) (Figure 3-Source Data 1). We therefore removed these modules from subsequent analysis. GM7 contains well characterised anterior markers including Otx2, Six3 and Pax6, whilst GM11 contains midbrain/hindbrain markers including Pax2, Nkx6-2 and Wnt4 (Figure 3-Source Data 1). We find that at ss4 and ss8 these gene modules are indeed upregulated in the forebrain/aPPR and midbrain/hindbrain, respectively (Figure 3-figure supplement 1A). To focus on our analysis on the segregation of neural, neural crest and placodal cells rather than on A-P patterning, A-P restricted modules were removed. The remaining five modules show broadly pan-neural (GM9), pan-placodal (GM23, GM21, GM24) or neural crest cell (GM13) expression at ss4 and ss8 (Figure 3A).

\section{Latent time analysis suggests late emergence of neural crest cells}

Given the cellular heterogeneity observed at early timepoints, discrete developmental stages do not necessarily accurately represent the developmental progression of individual cells towards a given fate. In contrast, latent time can measure the biological clock of individual cells as they differentiate and thus represents the order of cells along a developmental trajectory. To explore the expression dynamics of the five selected gene modules as neural, neural crest and placodal fates emerge, we first ordered cells across latent time using scVelo for the entire dataset (Figure 3B and Figure 3-figure supplement 1B). To investigate the segregation of all three fates, three separate trajectories need to be distinguished. To do this we calculated the probability of each cell to transit towards one of three terminal states (fate absorption probability) (Figure 3-figure supplement 1C). These probabilities were obtained using CellRank (Lange et al., 2022), which leverages RNA velocity and transcriptomic similarity information to predict transitions between cells. Mapping the fate probabilities of each cell onto UMAP plots of the full dataset (Figure 3C-E, see Figure 3B and Figure 2E-F for reference) shows that cells with low latent time values (i.e., early cells) have low probabilities of becoming neural, neural crest or placodal cells. As cells 'age' their fate probabilities increase as they become more transcriptionally like the terminal state. Unlike placodal and neural, neural crest fate probabilities are only high in 'late' cells (Figure 3B-C), reflecting the fact that defined neural crest cell clusters only appear at $\mathrm{HH} 7$. 

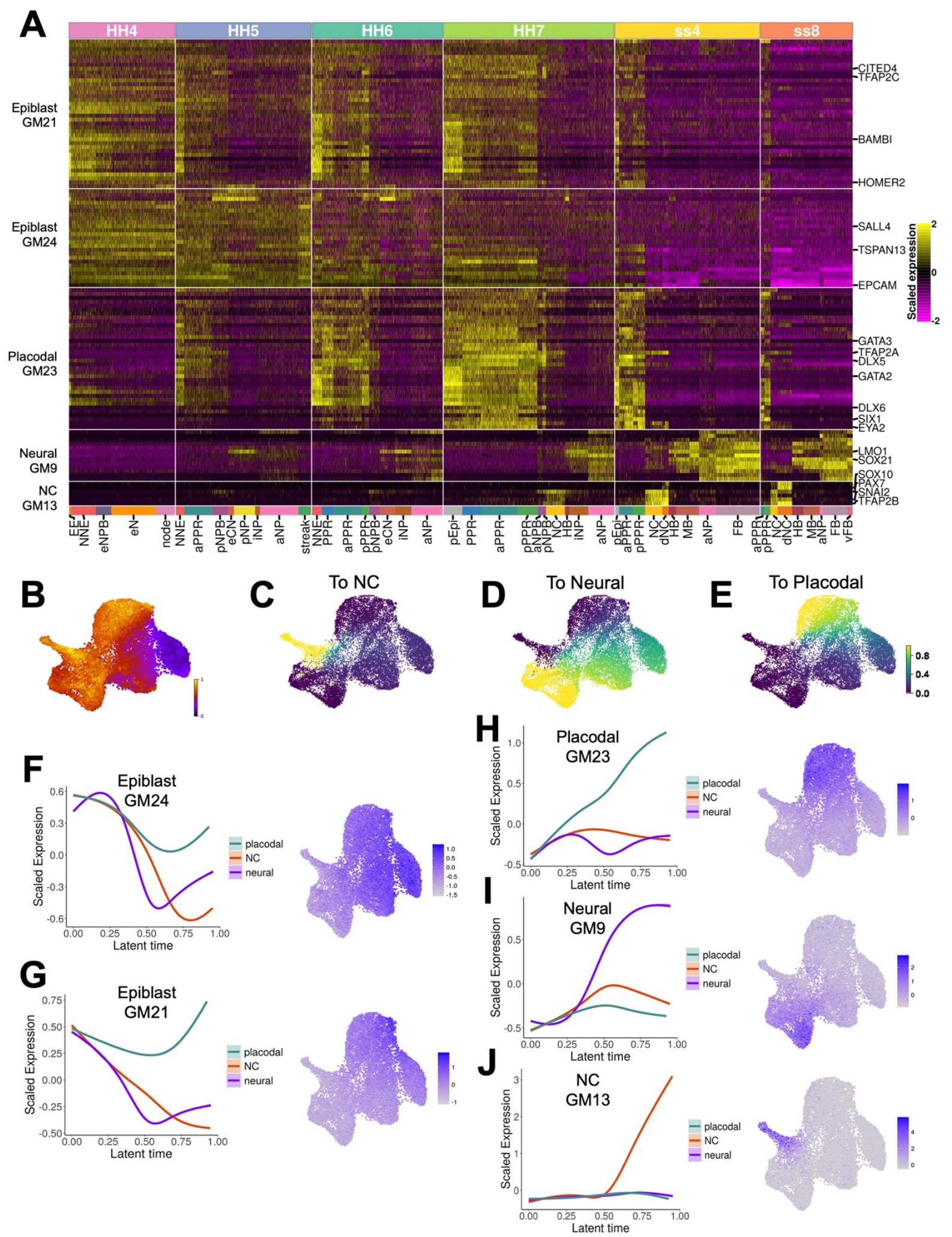

Figure 3. Gene module dynamics reveal key differences between the segregation of the PPR, neural crest and neural fates. (A) Heatmap of gene modules that display fate-specific expression (full list of gene modules available in Figure 3-Source Data 1). Gene modules were first unbiasedly filtered based on their differential expression between cell states (see methods and Figure 3-figure supplement 1A). Gene modules were further manually filtered based on 
expression patterns described in the literature (see results section: Defining neural, neural crest and placodal gene modules). Key genes of interest are highlighted on the right (for full gene list see Figure 3-Source Data 1). (B) UMAP plot of the full dataset (HH4-ss8) showing cell latent time values. (C-E) UMAP plots of the full dataset showing the fate absorption probabilities of each cell towards one of the three defined terminal states: neural crest, neural and placodal. (F-J) Gene module dynamics plots and feature plots for 5 gene modules: GM24, GM21, GM23, GM13 and GM9. Gene module dynamic plots display generalised additive models (GAMs) of scaled gene module expression across latent time. GAMs are weighted by the fate absorption probability of each cell towards one of the three terminal states (placodal, neural crest and neural). Feature plots show average scaled gene module expression on UMAPs of the full dataset.

Gene module dynamics reveals key differences in the activation of the transcriptional programmes associated to neural, neural crest and PPR specification

Having predicted the developmental trajectory of cells using scVelo and CellRank, we next modelled the expression of the five selected gene modules as a function of latent time with the aim to characterise their dynamics. Gene expression dynamics were modelled using generalised additive models (GAMs). GAMs were chosen because gene expression is highly dynamic and does not change linearly over time. For each gene module we fitted three separate GAMs, one for each fate (Figure 3F-J). The three fates were distinguished using the previously calculated fate absorption probabilities. This modelling approach reveals dynamic changes of gene expression. Three gene modules - GM9, GM13, GM23 - are initially inactive and are upregulated at different points across latent time in cells with neural, neural crest and placodal character, respectively (Figure $3 \mathrm{H}-\mathrm{J}$ ).

GM9 becomes upregulated in all neural clusters and includes neural markers like Lmo1 and Sox21 (Figure 3A, I; Figure 3-Source Data 1). Although the expression of different genes within this module correlates broadly, there is some gene-gene variation. For example, Lmo1 is the first gene to be expressed and exhibits highly dynamic expression over time (Figure 3A). It is first found at $\mathrm{HH} 5$ within the eCN, pNP and iNP, before being upregulated across the entire neural plate at $\mathrm{HH} 7$ and ss4. At ss8, Lmo1 is specifically downregulated within the MB. In contrast, Sox 21 is first upregulated at $\mathrm{HH} 5 / 6$ and is consistently expressed throughout all neural cell clusters (Figure 3A).

In contrast, modelling reveals that GM13 is upregulated in cells with neural crest identity (Figure 3J). Indeed, it contains well known neural crest markers Pax7, Snai2, Sox10 and Tfap2b (Figure 3A, Figure 3-Source Data 1) and is initially broadly activated in the NPB and neural crest clusters at $\mathrm{HH} 7$. At ss 4 and ss8, this module remains strongly expressed within 
neural crest and delaminating neural crest clusters (Figure 3A). Within GM13 individual genes are activated at different time points: Pax7 is the first gene to be expressed in the pNPB at $\mathrm{HH}$, while Sox10 starts to be expressed only from ss4 onwards.

Finally, modelling the expression dynamics of GM23 shows that it is activated early and becomes upregulated in cells with placodal character across latent time (Figure $3 \mathrm{H}$ ). This prediction is supported by the fact that GM23 is highly expressed in PPR clusters at ss 8 and contains bona fide placodal genes like Six1 and Eya2 (Figure 3A, Figure 3-Source Data 1). At $\mathrm{HH} 4$, most GM23 genes are not expressed, but are broadly activated at HH5 in PPR, nonneural and NPB clusters, where they remain active until becoming restricted to PPR cells at ss4 (Figure 3A). Comparing the onset of activation of these three gene modules across latent time reveals a clear temporal order with the placodal module being activated first, followed by the neural and neural crest gene modules.

In contrast to these modules, modelling of GM24 and GM21 shows that they are initially expressed in all three cell populations before being rapidly downregulated within neural crest and neural cells (Figure 3F, G). Indeed, genes in GM24 are broadly expressed across the epiblast at $\mathrm{HH} 4$, and then become gradually restricted to PPR, non-neural and NPB clusters at $\mathrm{HH} 6 / 7$, and to PPR cells at ss4/8 (Figure 3A). GM21 displays similar dynamics but is restricted to non-neural clusters somewhat earlier (HH5; Figure $3 \mathrm{~A})$. Although some genes within these epiblast modules have been described as non-neural/PPR specific based on in situ hybridisation (Tfap2c, Bambi, Homer2, Tspan13) (Bell et al., 2004; Hintze et al., 2017; Mehdizadeh et al., 2021; Reichert et al., 2013; Rothstein \& Simoes-Costa, 2020), others are found to be expressed within the early neural territory (Epcam, Sall4, Cited4) (Barembaum \& Bronner-Fraser, 2007; Bell et al., 2004; Trevers et al., 2021) (Figure 3-Source Data 1).

In summary, modelling the expression dynamics of selected gene modules provides new insight into the sequential activation of fate-specific developmental programmes, as well as proposing key differences in the mechanism by which neural, neural crest and placodal cells are specified. We show, using an unbiased gene module approach, that a placodal gene module is activated first, followed by neural and neural crest modules. While neural and neural crest modules are specifically activated in the relevant cell clusters alone, two modules that are later restricted to the PPR are initially broadly expressed across the epiblast. We therefore propose that the specification of neural and neural crest states may require the downregulation of epiblast modules which later become confined to placodal cells. 


\section{Redefining the NPB cell state}

NPB cells are thought to retain the potential to give rise to neural, neural crest and placodal lineages, and recent single cell analysis suggests that they can do so even at late neurulation stages (Roellig et al., 2017; Williams et al., 2022). So far, our analysis reveals an early NPB cell population identified by the overlapping expression of non-neural and early neural markers at primitive streak stages, albeit being transcriptionally highly heterogeneous. In contrast, Msx 1 and Pax7 which have previously characterised as NPB specifiers, are only upregulated at the NPB at head process stage, have posterior character and are largely absent from placode precursors (Figure 1E, F, Figure 1-figure supplement 2B, C). At ss4, NPB cells can no longer be identified based on the expression of these specifier genes, yet we find continued presence of bridge cells that connect neural, neural crest and placodal cell clusters. Thus, among the key questions remaining are whether NPB cells have a unique transcriptional profile and whether bridge cells have NPB character with the potential to give rise to multiple fates.

To explore this idea, we focused on the decision between neural crest and placode precursors. Neural crest and placode specific transcription factors cross-repress each other while enhancing their own expression (for review: Thiery et al., 2020). It is therefore likely that coexpression of such genes may cells which are still plastic. We therefore examined the coexpression of neural crest and placode specific gene modules in a subset of cells composed of neural crest, placodal and NPB cell states between HH5-ss8 (for workflow explanation see Figure 3-figure supplement 2).

Defining neural crest and placode specific gene modules

First, we sought to identify gene modules that are specifically restricted to neural crest and placode cells at late stages. GM analysis in cells collected from ss8 identified 15 modules (Figure 4 and Figure 4-Source Data 1) that are differentially expressed in at least one cell state. GM9-13 and GM16-17 are characteristic for neural cells with different A-P identities. Seven gene modules are absent from neural populations but expressed in placodal cells (GM4-6), neural crest (GM1-2) or both (GM7-8). GM5 contains well characterised placodal markers including Six1, Homer2 and Znf385c (Figure 4-Source Data 1) and is expressed in both aPPR and pPPR clusters. This module was therefore selected as a pan-placodal module. GM2 is expressed in both neural crest and delaminating neural crest clusters (Figure 4; NC, dNC), contains neural crest specifiers like FoxD3, Snai2, Sox9, Pax7 and Msx1, as well as

512 the Wnt ligands Wnt1 and Wnt6 (Figure 4-Source Data 1) and thus represents a bona fide neural crest specific module. Although GM1 contains multiple neural crest markers (Ets1, 
bioRxiv preprint doi: https://doi.org/10.1101/2022.02.15.480567; this version posted February 16, 2022. The copyright holder for this preprint (which was not certified by peer review) is the author/funder, who has granted bioRxiv a license to display the preprint in perpetuity. It is made available under aCC-BY-NC-ND 4.0 International license.

Sox10, Sox8; Figure 4-Source Data 1), it is only expressed in delaminating neural crest cells (Figure 4) and was therefore excluded for further analysis. Thus, GM5 and GM2 were considered as pan-placodal and pan-neural crest module and used for co-expression analysis.

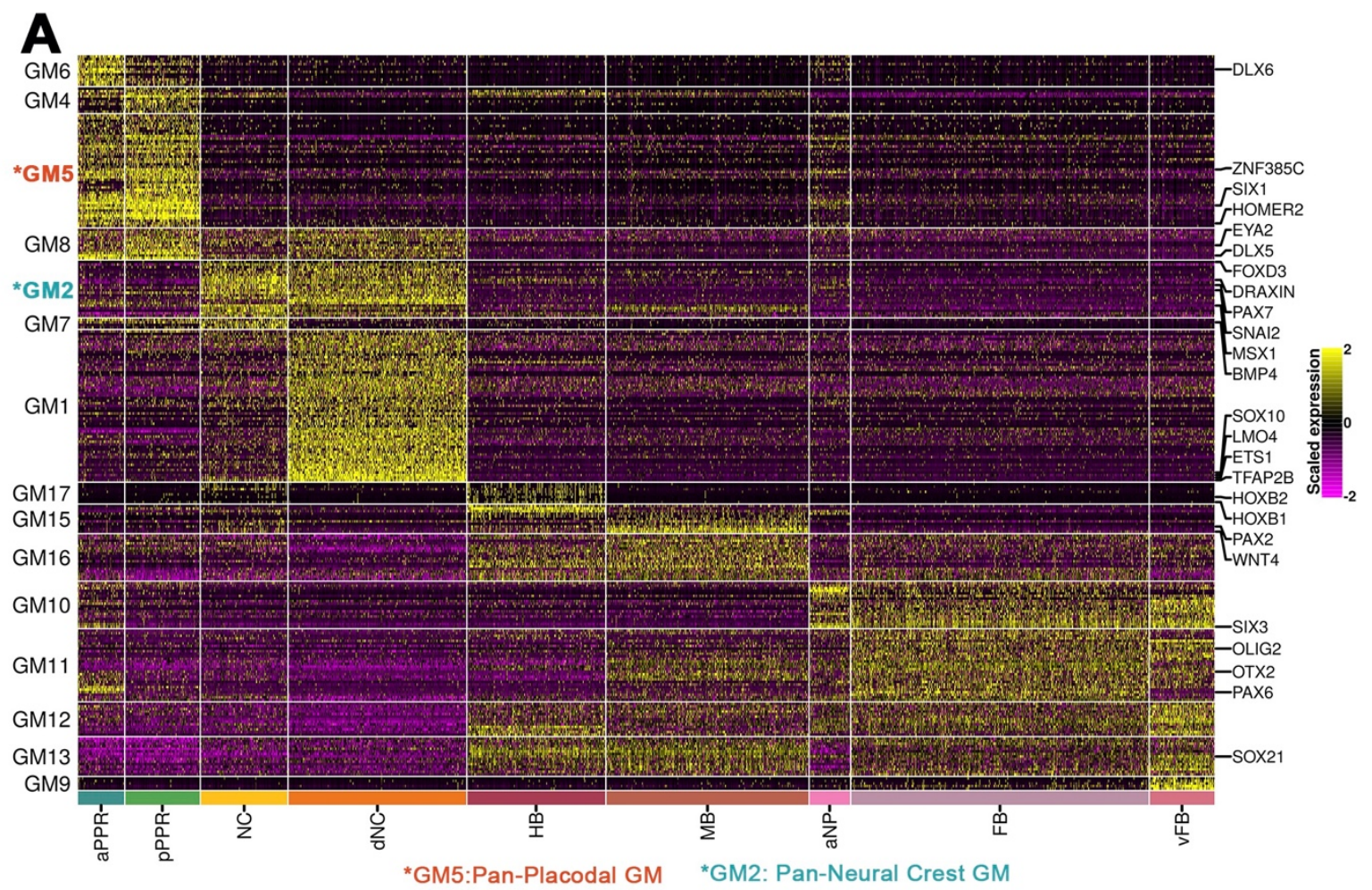

Figure 4. Gene modules calculated at ss8. (A) Heatmap showing gene modules calculated from ss 8 cells and then filtered to include only those that are differentially expressed between cell states (see methods; full list of gene modules available in Figure 4-Source Data 1). GM5 and GM2 are highlighted as the selected pan-placodal and pan-neural crest gene modules used for subsequent analysis in Figure 5.

\section{Placodal and neural crest bridge cells exhibit NPB-like co-expression}

To investigate the co-expression of these modules in NPB cells and their derivatives, we first selected NPB (aNPB, pNPB), neural crest (NC, dNC) and PPR (aPPR, pPPR, PPR) cell states from HH5-ss8 (Figure 5A, red cells; thereafter referred to as NPB subset). Next, we reclustered this subset of cells based on the expression of all genes (Figure 5B). UMAP analysis suggested a bifurcation of neural crest and placodal fates taking place between $\mathrm{HH} 7$ and ss4, with NPB cells located at the boundary between neural crest and aPPR cell clusters (Figure $5 B, C)$.

To examine the relative expression of pan-placodal and pan-neural crest modules across the entire cell subset, we ordered cells according to the expression ratio of both modules and 
grouped them into 10 evenly sized bins (Figure 5D and Figure 5-figure supplement 1 ). Cells at the extreme ends exclusively express the pan-neural crest (Bin 1) or the pan-placodal gene module (Bin 10). As expected, these cells represent mostly delaminating neural crest or placodal cells, respectively. However, cells in Bin 3 show equal expression levels of both modules and contain a mixture of NPB, PPR and neural crest cell states (Figure 5D). We then mapped Bin 3 cells back to the NPB subset UMAP (Figure 5B) and find that they indeed correspond to NPB cells located specifically between PPR and neural crest cell clusters (Figure 5E). This contrasts with Bin 1 and 10 cells, which map to the opposite ends of the UMAP and their respective fates (Figure 5E). Thus, most cells expressing intermediate levels of both neural crest and placodal gene modules are classified as NPB.

Bin 3 cells contain a mixture of cells collected from HH5-ss8. To evaluate which cell states express similar levels of pan-neural crest and pan-placodal modules at different developmental times, we mapped Bin 3 cells back to the UMAPs of individual stages (Figure 5G). At $\mathrm{HH} 5$ and $\mathrm{HH} 6$, neural crest cell states are not defined yet (Figure 5F colour coded according to Figure 1B-D and Figure 2A-C), and Bin 3 cells consist of a mixture of aPPR and pNPB cells (Figure 5F, G). At HH7, Bin 3 contains almost no PPR cells, instead these cells are mostly neural crest and NPB (Figure 5F, G). At ss4, the number of cells expressing similar levels of the pan-placodal and pan-neural crest modules decreases sharply. Although at this stage a NPB state based on the expression of NPB specifiers cannot be classified, Bin 3 cells are found at the boundary between neural crest and PPR clusters. In fact, they represent the bridge cells predicted to be in an undetermined transcriptional state by RNA velocity (Figure $2 G$ ). Once placodal and neural crest lineages are largely segregated at ss8, only very few scattered cells express similar levels of pan-neural crest and pan-placodal modules (Figure $5 G)$.

The analysis above only focuses on cells of the NPB subset expressing pan-placodal and panneural crest modules at a similar level, which are likely to be transcriptionally indeterminate and not yet committed to either of the two fates. To identify cells with varying levels of coexpression and those that may transit to either fate, we visualised cells that co-express these modules at different levels. We calculated pan-neural crest and pan-placodal module coexpression per developmental stage and filtered cells below a minimum threshold of 0.3 (Figure $5 \mathrm{H}$ ). At HH5/6 almost all cells classified as NPB and placodal cells co-express both modules above the threshold. However, this changes at $\mathrm{HH} 7$, when co-expressing cells are largely restricted to the NPB and neural crest clusters (Figure $5 \mathrm{H}$ ). By ss4, they are only found in bridge cells between placodal and neural crest clusters (Figure 2G). 
574 Given the different proportions of cells co-expressing these modules at each stage, we 575 investigated whether the distributions in co-expression levels varied between them (Figure 5I).

576 From the early to late stages, the distribution gradually changes from a normal distribution

577 towards a negative binomial distribution. This suggests that at $\mathrm{HH} 5-\mathrm{HH} 7$ cells co-express pan-

578 neural crest and pan-placodal modules at similar levels, while only a small proportion of co-

579 expressing cells express these modules at high levels later.

580

581 Our analysis proposes that NPB cells are transcriptionally indeterminate, co-expressing gene 582 modules that characterise both neural crest and placodal cells. This expression profile is likely 583 to endow them with the potential to give rise to both, if not all NPB fates. The proportion of 584 these cells decreases over time, but bridge cells at ss4 retain this characteristic and are 585 therefore likely to retain bi- or multipotency even as the neural tube closes. 

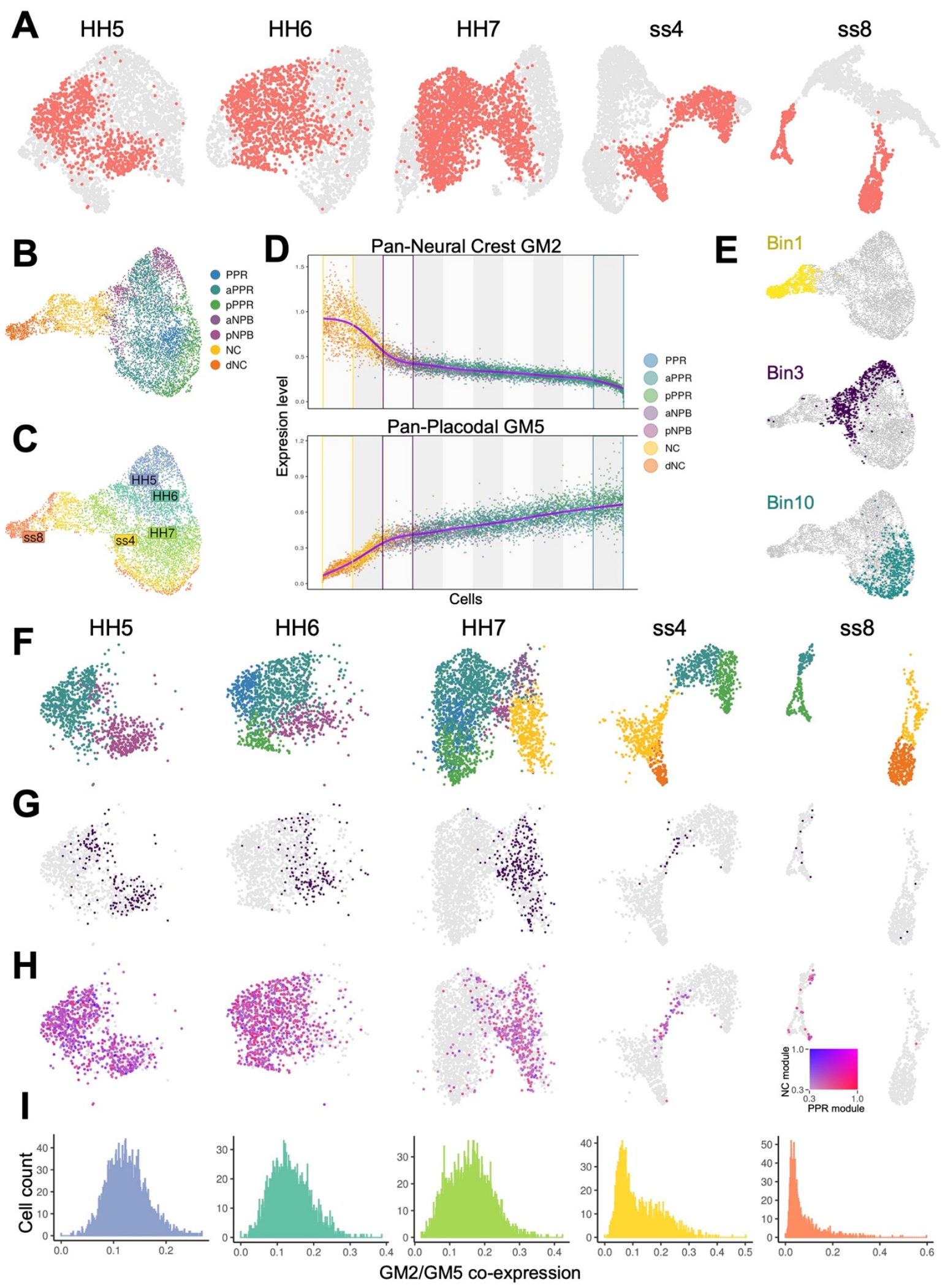

Figure 5. Cells at the NPB co-express placodal and neural crest gene modules. (A) UMAP plots of from each stage with all PPR, neural crest and NPB cells highlighted in red, which were selected as the 'NPB subset'. (B) UMAP plot of the NPB subset coloured by cell state. (C) UMAP plot of the NPB subset coloured by developmental stage. (D) Average 
normalized expression of placodal and neural crest gene modules (GM5 and GM2, respectively) identified at ss8 (see Figure 4). Cells are ordered by their expression ratio of these two modules and coloured by cell state. Cells were grouped into 10 evenly sized bins (background shaded regions). Three bins (1,3 and 10) are overlaid to highlight cells with high neural crest/low placodal (bin 1), equivalent levels of both (bin 3) and high placodal/low neural crest (bin 10) gene expression. (E) UMAP plots of the NPB subset displaying the cells captured within bins 1, 3 and 10. (F-H) UMAP plots from each developmental stage, displaying only cells in the NPB subset (equivalent to red cells in A). (F) Cells are coloured by cell state. (G) Cells which are captured in bin 3 are highlighted. (H) Co-expression analysis of the panneural crest and pan-placodal modules (see methods). Cells which co-express both modules above 0.3 are coloured. (I) Histograms revealing the distribution of co-expression of the panneural crest and pan-placodal modules at each developmental stage. At later stages (ss4ss8), the distribution of co-expression shifts from a normal to a negative binomial distribution.

\section{A temporal hierarchy of gene module expression in neural crest and placode precursors}

To investigate the mechanism by which NPB cells segregate into the neural crest and placodal fates, we modelled gene module dynamics across the NPB subset alone (for workflow explanation see Figure 3-figure supplement 2). Previous modelling dynamics (Figure 3) analysed gene modules across the whole dataset to uncover sequential activation of placodal, neural and neural crest gene programmes at higher level. In contrast, focusing on the NPB subset provides a higher gene module resolution and consequently allows modelling of multiple placodal and neural crest gene modules and establishing the order in which different genes are activated.

As before, we first defined gene modules characteristic for neural crest and placodal cells. Gene modules were calculated using the neural crest, placodal and NPB cell state subset from HH5-ss8 as described above. Only 10 modules with differential expression between the neural crest and placodal fates were considered. These were filtered further to include only those that contained genes defined in the binary knowledge matrix as present in neural crest, delaminating neural crest or PPR cell states. This analysis identified three modules (GM1214) upregulated in placodal and four in neural crest cells (GM40 and GM42-44) between $\mathrm{HH} 7$ and ss8 (Figure 6E, Figure 6-Source Data 1).

To predict cell trajectories towards neural crest or placodal terminal states prior to GM modelling, we performed scVelo analysis on the NPB subset (Figure 6B-D). As before, we used velocity and CellRank to calculate latent time and determine the probability that each cell 
will become neural crest or placodal (Figure 6A-D). In turn, latent time and fate probabilities were used to model gene module dynamics (Figure 6F-H).

Of the placodal modules, GM14 and GM12 are expressed as early as HH5. GM12 is expressed throughout both aPPR and pNPB and contains early non-neural/PPR markers like Gata2/3 (Figure 6E, Figure 6-Source Data 1). At HH6, GM12 is downregulated in the NPB and remains confined to the PPR clusters until ss8. Unlike GM12, GM14 is initially restricted to the PPR and only activated in the NPB at $\mathrm{HH} 7$. At ss 4 and ss8 its expression is confined to the PPR, with a higher expression in anterior cells. This module contains pan-placodal genes like DIx5/6 as well as anterior markers Six3 and Pax6 (Figure 6E, Figure 6-Source Data 1). GM13 is the last placodal module to be activated and contains the definitive placodal transcripts Six1 and Eya2. It is first expressed at low levels at $\mathrm{HH} 6$ before being broadly activated across the PPR at $\mathrm{HH} 7$ through to ss8. We also observe weaker expression of this module within NPB cells at $\mathrm{HH} 7$ (Figure 6E). To visualise the sequential activation of these modules across latent time, we used the same approach described above (Figure 3F-J), whereby gene expression of each cell was weighted based on its placodal fate probability. This analysis shows that GM12 is activated before GM14 and GM13 in latent time (Figure 6F). Whilst the expression of GM12 and GM14 does not change dramatically, GM13 is highly upregulated over latent time within the placodal cells (Figure 6F).

The four neural crest gene modules each contain bona fide neural crest transcriptional regulators including Msx1, Zic1, Zeb2, Lmx1b (GM40), Pax7, Snai2, Axud1, Foxd3, Tfap2b, Sox9 (GM42), Sox5, Id1/2 (GM44) and Sox10, Ets1, Lmo4 and Sox8 (GM43) (Figure 6E and Figure 6-Source Data 1). Interestingly, our analysis groups these factors unbiasedly into four modules, and visualisation of their expression across latent time weighted by neural crest fate probability shows a clear sequential activation from GM40 to GM42, GM44 and GM43 (Figure 6G). GM40 is activated first, initially broadly across aPPR and pNPB clusters at $\mathrm{HH} 5$ and $\mathrm{HH} 6$, before becoming restricted to neural crest and NPB clusters from $\mathrm{HH} 7$ onwards. By ss8, it is downregulated in the delaminating neural crest population. GM42 and GM44 are highly upregulated only in neural crest and delaminating neural crest cells, with GM42 first expressed at $\mathrm{HH} 7$ and GM44 at ss4. Lastly, GM44 is activated specifically within the delaminating neural crest cells at ss 4 where it is further upregulated at ss8 (Figure 6E). This order suggests a hierarchical relationship between these factors in the neural crest gene regulatory network. 

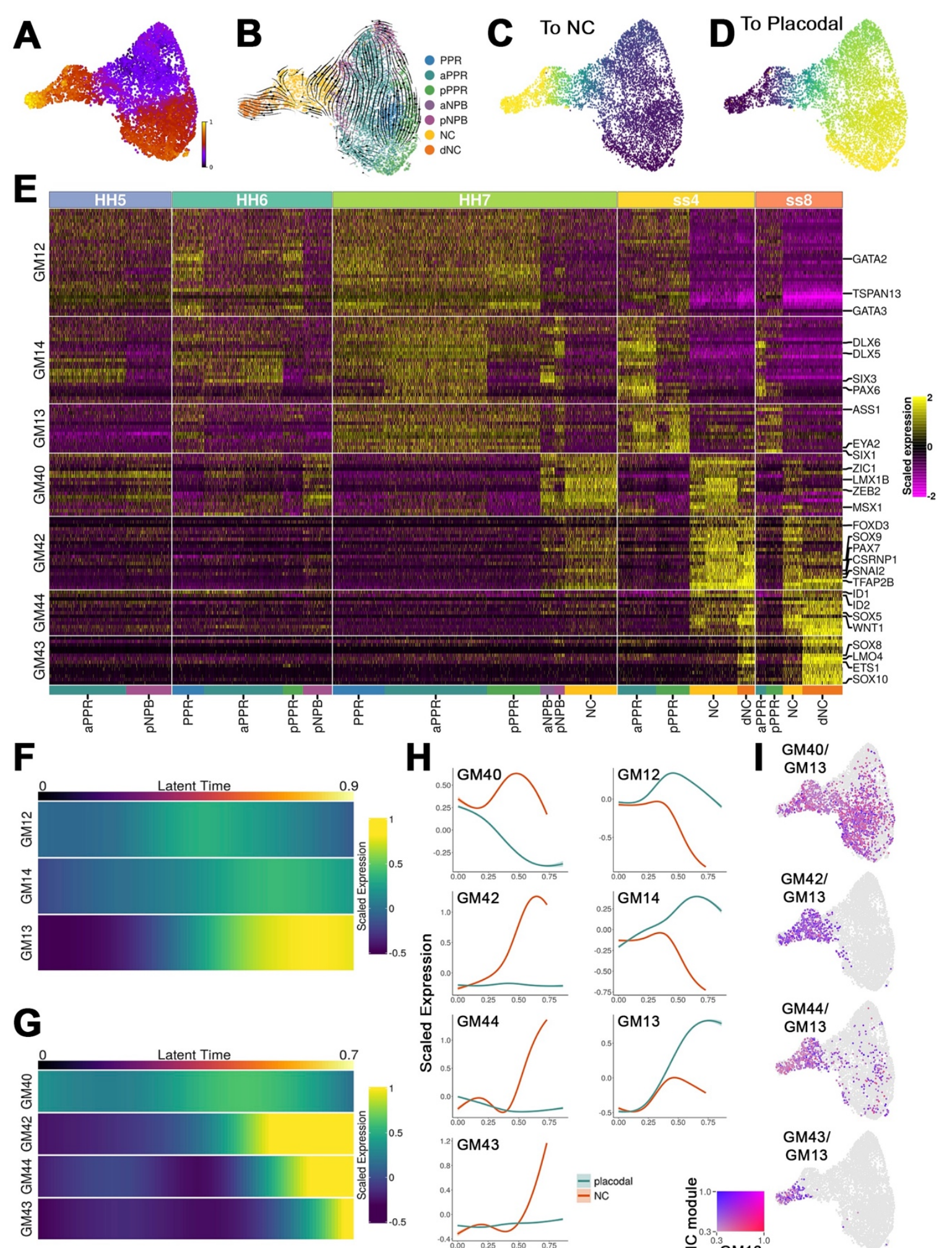

H
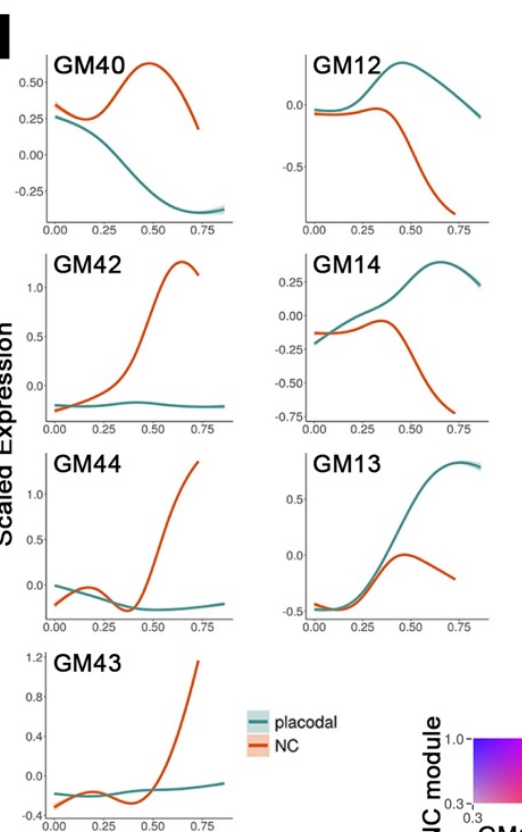

Latent time

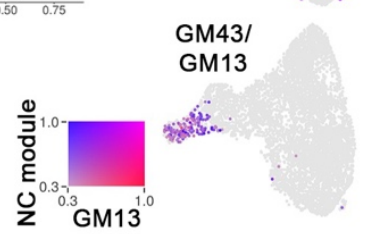

Figure 6. Co-expression analysis suggests neural crest specification requires downregulation of placodal genes. (A) UMAP plot of the NPB subset with cells coloured by latent time. (B) UMAP plot with cells coloured by cell state classifications and overlaid with RNA velocity vectors depicting the predicted directionality of transcriptional change. (C-D) UMAP plots showing the fate absorption probabilities of each cell towards the specified 
terminal states (placodal or neural crest). (E) Heatmap showing gene modules calculated across the NPB subset (full list of gene modules available in Figure 6-Source Data 1). To identify gene modules potentially involved in placodal or neural crest specification, gene modules were filtered to include those that show differential expression between placodal and neural crest cell states (see methods). Genes specified as neural crest or placodal in our binary knowledge matrix (Supplementary File 1) were selected as bait genes to further filter the gene module list for visualisation. (F) Heatmap displaying predicted scaled expression of placodal gene modules (GM12, GM13 and GM14) within the placodal lineage across latent time. Gene expression was predicted by fitting a GAM which models scaled gene expression as a function of latent time. Gene expression was weighted in the GAM using the placodal fate lineage absorption probability. (G) Heatmap displaying predicted scaled expression of neural crest gene modules (GM40, GM42, GM43 and GM44) within the neural crest lineage across latent time. Gene expression was modelled as in $(F)$ but using neural crest fate absorption probabilities to weight gene expression. (H) Gene module dynamics plots for all 7 gene modules: GM40, GM42, GM44, GM43 (neural crest) and GM12, GM14, GM13 (placodal). Gene module dynamic plots display GAMs of scaled gene module expression across latent time calculated as in $(\mathrm{G})$ and $(\mathrm{H})$. GAMs were weighted by the fate absorption probabilities of each cell towards either placodal or neural crest terminal states.

While gene module selection was based on prior knowledge of marker genes, this analysis also allows us to predict novel factors with similar expression profiles. These include Nfkb1, Ass 1 and Fam184b in placodal cells and Enc1, Rftn2 and Olfm/3 in neural crest clusters. Modelling their expression across latent time reveals highly conserved dynamics with known factors (Figure 6-figure supplement 2), thus raising the possibility that they may play a role in placode and neural crest cell specification.

In summary, analysing gene module dynamics across the NPB subset identifies reveals the sequential activation of different co-regulated genes in placodal and neural crest cell. Many genes within these modules have been previously characterised and are known to form part of the neural crest or placode gene regulatory network (for review: Grocott et al., 2012; Pla \& Monsoro-Burq, 2018; Schlosser, 2006, 2014; Simoes-Costa \& Bronner, 2015; Thiery et al., 2020). This unbiased approach groups these factors and predicts a hierarchical order, as well as identifying new candidate factors which may play important roles in the segregation of the neural crest and placodal fates (Figure 6-figure supplement 2).

\section{Emergence of neural crest cells may require the downregulation of definitive placodal} genes

Various factors within the placodal and neural crest gene modules have previously been implicated in conferring neural crest and placodal identity. To assess their temporal activation, 
711 we modelled their dynamics across both developmental trajectories simultaneously and assessed their co-expression in individual cells.

As with the previous analysis (Figure 3F-J) we found that gene modules varied in their expression dynamics across both fates over latent time. Of the neural crest modules, GM40 (containing Msx1 and Zic1) is the first to be activated in both the neural crest and placodal cells before being downregulated in placode precursors at $\mathrm{HH} 6 / 7$ (Figure 6E, $\mathrm{H}$ ). In contrast, GM42, GM44 and GM43 are exclusively upregulated in the neural crest lineage (Figure 6H). Of the placodal modules, GM12 and GM14 are initially highly expressed in both cell types and then downregulated in the neural crest lineage. In contrast, GM13 is not initially expressed, but starts to be upregulated in both neural crest and placodal cells as time progresses, before being rapidly downregulated in neural crest cells as its expression increases in placode precursors. Interestingly, GM13 contains definitive placodal markers Six1 and Eya2 which in turn repress neural crest factors including Pax7 (Brugmann et al., 2004; Christophorou et al., 2009). Its initial upregulation within the neural crest cells suggests that their specification requires the downregulation of this module. In contrast to GM13, placodal cells never upregulate definitive neural crest modules (GM42-44).

Since neural crest precursors initially appear to express the late placodal module GM13, we sought to investigate which cells co-express GM13 with each of the four neural crest modules. As expected from the expression of the early neural crest module GM40 in placodal precursors, we find broad co-expression of both modules (Figure 6I) within neural crest and placodal cell clusters both, at the bifurcation point and in segregated clusters. In contrast, the later neural crest modules are not co-expressed with GM13 extensively within the placodal branch. However, we do find co-expression within the neural crest branch. This suggests that some residual placodal gene expression remains in the neural crest clusters even at ss8. This supports our previous finding that the specification of the neural crest state may require the downregulation of placodal gene modules (Figure 3). 
A

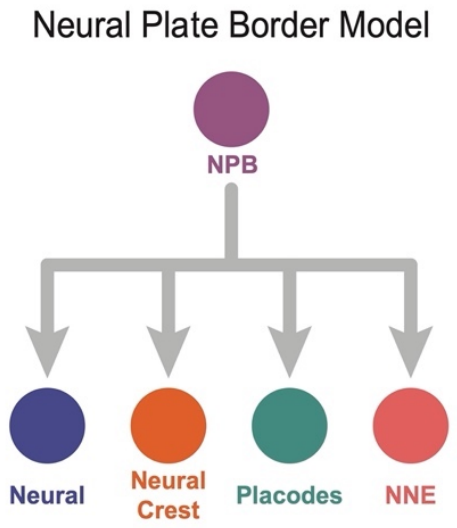

B

Dual Competence Model

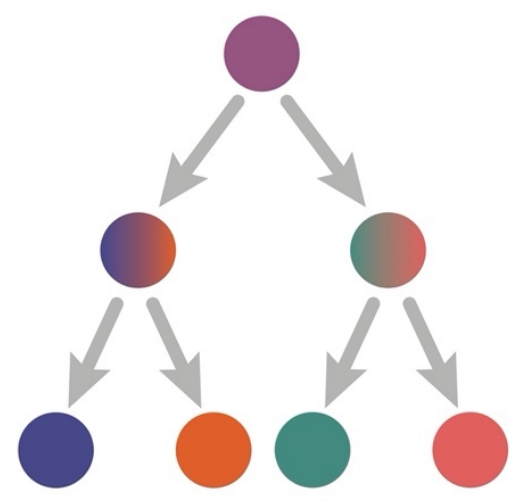

C

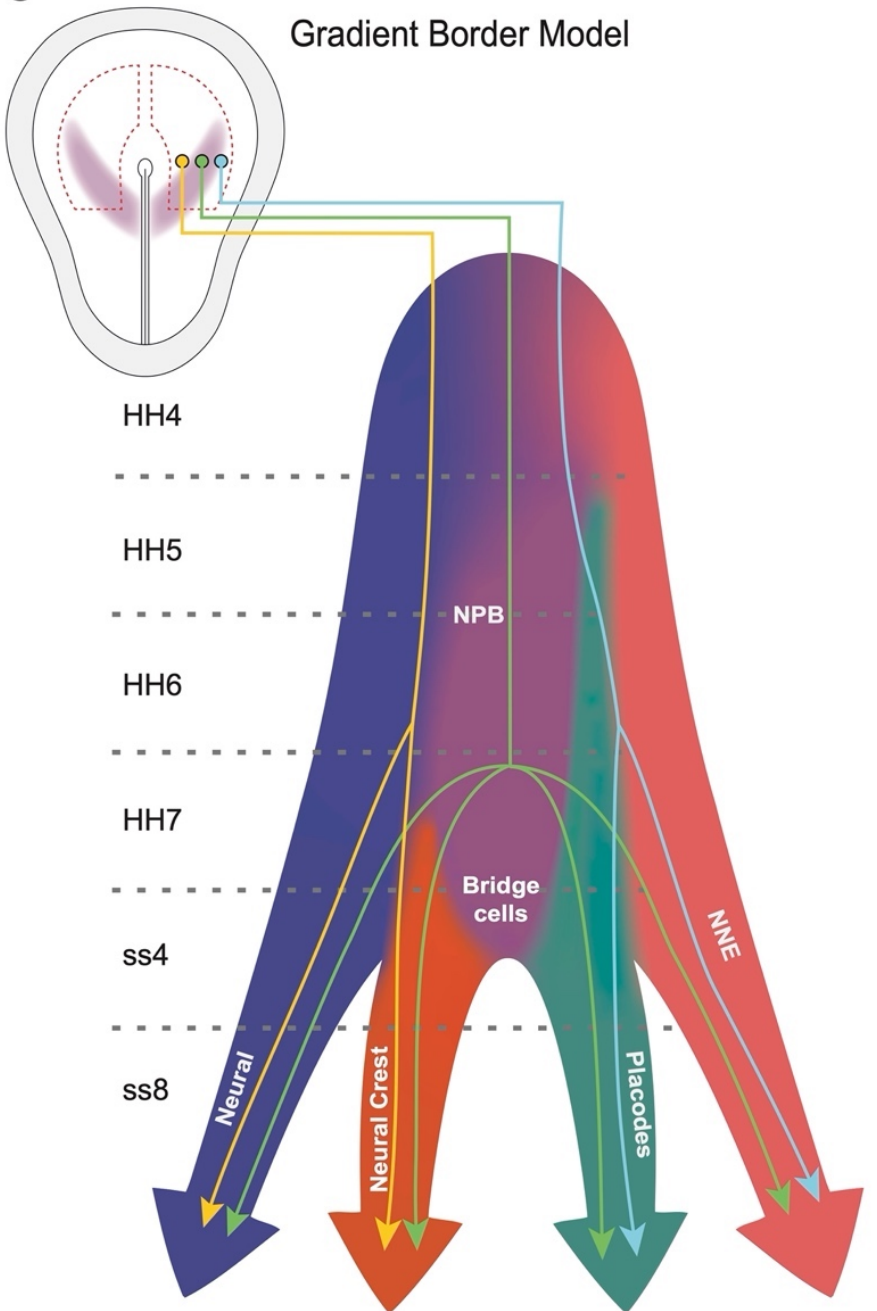

Figure 7. Gradient border model resolves conflicting models of lineage segregation at the neural plate border. (A) Schematic illustrating the neural plate border model. This model illustrates that cells at the neural plate border are multipotent and able to give rise to neural, neural crest, placodal and non-neural ectoderm (NNE) lineages. (B) Schematic illustrating the dual competence model. This model suggests that the neural plate border is first defined into non-neural/placodal and neural crest/neural competence domains prior to further subdivision into each of the final four cell lineages. (C) Schematic illustrating the gradient border model. This model proposes a probabilistic model of cell fate allocation which is intrinsically linked to the spatiotemporal positioning of a cell. We suggest that cells located within the medial neural plate border (yellow cell lineage) will give rise to neural and neural crest, cells within the lateral neural plate border (blue cell lineage) to non-neural ectoderm and placodes, whilst subsets of cells (including bridge cells) will remain in an indeterminate state and continue to give rise to all four lineages even at late stages of neurulation (green cell lineage). Shaded background colours represent the cell state space. 


\section{Discussion}

The regulation of cell fate specification at the NPB has been extensively studied in a range of model systems, giving rise to a wealth of knowledge regarding transcriptional regulators and their interactions. Nevertheless, our understanding as to how individual cells undergo cell fate decisions has been limited by our inability to study transcription and gene regulation at a single cell level. Recent studies have demonstrated that in contrast to what was previously thought, cells co-express markers that specify either placodal, neural or neural crest fates, even during late neurulation (Roellig et al., 2017; Williams et al., 2022). This newly characterised cellular heterogeneity requires us to revisit models for cell fate specification at the NPB and reconsider our definition of the NPB cell state itself.

Our study unbiasedly characterised ectodermal cell states and cellular heterogeneity from primitive streak stages though to late neurulation in chick. We demonstrate that although significant transcriptional differences are apparent between cells at primitive streak stages, due to high levels of heterogeneity, cells do not cluster into discrete cell states exhibiting restricted expression patterns until the 1-somite stage. Instead, cell states are initially characterised by the overlapping expression of broad early neural and non-neural genes, as well as axial patterning markers. We identify increased cell state diversification during neurulation, and segregation of neural, neural crest and placodal lineages taking place across 1-somite to 8-somite stages. High resolution transcriptional analysis of cells at the NPB reveals that by the 4-somite stage, placodal and neural crest lineages have largely segregated. However, despite this segregation, we continue to identify high levels of co-expression of placodal and neural crest genes within bridge cells which are predicted to be in a dynamic transcriptional state.

Specification of neural and neural crest cell lineages requires downregulation of broad epiblast modules

Although specification networks for the neural, neural crest and placodal lineages are well established (for review: Betancur et al., 2010; Grocott et al., 2012; Pla \& Monsoro-Burq, 2018; Stundl et al., 2021; Thiery et al., 2020), the order in which these lineages are specified remains unclear. Our unbiased gene module analysis has identified groups of genes which correlate and are specifically upregulated within each cell fate. Our gene modules support the specification networks established in previous studies. Pax7, Tfap2b, Snai2 and Sox10 cluster together and are upregulated in neural crest states, while Six1, Eya2, Gata2/3 and D/x5/6 are 
We also find putative novel regulators of these cell states, as well as the order in which these cell fates are specified. We note the sequential temporal activation of placodal, neural and neural crest specific gene modules, suggesting that these states are specified in that order. However, there is also asynchrony in the initiation of cell specification; although we identify placodal cells as early as $\mathrm{HH} 5$, we also observe indeterminate NPB cells which are only later specified. We suggest cell fate allocation at the NPB is a gradual process, with the NPB narrowing over time as cells within this population are specified.

Alongside characterising placodal, neural and neural crest gene modules, we observe the expression of two modules which exhibit initial broad pan-epiblast expression at primitive streak stages, but which are confined to placodal cell states at the 4 and 8-somite stages. Within these epiblast modules, unsurprisingly we note the expression of important placodal and non-neural genes Tfap2c, Homer2 and the BMP inhibitor Bambi. Alongside these markers, we also find the pluripotency marker Sall4 and Tspan13, with the latter shown to be positively regulated by Six1 (Mehdizadeh et al., 2021). A recent study in chick suggests that based on transcriptional profiling a 'pre-border' state can already be defined prior to gastrulation. In vitro experiments show that the pre-gastrula epiblast is specified as NPB; once cells have acquired a 'pre-border' state they can become neural and placodal precursors depending on the signalling input (Trevers et al., 2018). Given that the expression the epiblast modules becomes confined to the placodes, we speculate that the specification of the neural crest and neural states requires the downregulation of these modules.

Redefining the neural plate border based on the co-expression of competing gene modules

Members of Zic, Tfap2, Pax, Msx and Dlx transcription factor families have been termed NPB specifiers (for review: Stundl et al., 2021; Thiery et al., 2020). Tfap2a has been identified as a pioneer factor which triggers the expression of other NPB specifiers and is required but not sufficient for both neural crest and placodal cell fates (Bhat et al., 2013; Rothstein \& SimoesCosta, 2020). The cell fate switching role of Tfap2a depends upon its association with its cofactors, Tfap2b and Tfap2c. Tfap2c is necessary for the induction of NPB specifiers Ms $x 1$, Pax7 and Zic1, whilst Tfap2b is later required for downstream neural crest specification (Rothstein \& Simoes-Costa, 2020).

Gene module analysis across the neural plate border highlights the sequential activation and restriction of previously characterised NPB specifiers to neural crest cells. Early NPB specifiers Msx1 and Zic1 are initially broadly expressed across pNPB and aPPR cells at HH5, 
but later become downregulated in the placodal lineage and upregulated in the neural crest. The gene module containing $P a x 7$ is activated slightly later and contains other NPB specifiers Axud1 and Tfap2b, as well as definitive neural crest markers FoxD3 and Snai2. Unlike the Msx1 module, which is initially upregulated in both aPPR and pNPB clusters at HH5, the Pax7 module is specifically restricted to the NPB and subsequent neural crest clusters (Figure 6F, $\mathrm{H}$, and Figure 6-figure supplement 1 ). This analysis reveals that despite these markers having been previously characterised as NPB specifiers, their expression becomes restricted to the neural crest. In contrast, $D / \times 5 / 6$ and Tfap2a are both found within a gene module that is later restricted to the placodes (Figure $3 \mathrm{~A}$ ).

Although Tfap2a is grouped in the same module as placodal specifiers Six 1 and Eya2, it is expressed in both placodal and neural crest cell states, even at later stages. This expression reflects the key role that Tfap2a plays as a pioneer factor in both neural crest and placodal specification, however its expression alone is not sufficient to classify the NPB state. At $\mathrm{HH} 5$, although Tfap2a is expressed within the pNPB cluster alongside other NPB specifiers, it is also expressed within aPPR cells which never appear to be exposed to NPB specifiers including Pax7, Pax3 and Tfap2b (Figure 1E).

To classify the NPB as a discrete state, NPB markers need to be uniformly upregulated in NPB cells which give rise to neural, non-neural ectoderm, neural crest and PPR fates. Given the non-uniform expression of previously characterised NPB specifiers (Basch et al., 2006; Ezin et al., 2009; Hong \& Saint-Jeannet, 2007; McLarren et al., 2003; Rothstein \& SimoesCosta, 2020; Streit \& Stern, 1999), and the expression of Tfap2a in both the NPB and early aPPR cells which are unlikely to give rise to other fates, we propose that the NPB state cannot be accurately classified using just these previously established genes. Instead, we suggest that the NPB is better defined by the co-expression of markers regulating alternative cell fates.

\section{Gradient border model resolves the neural plate border and binary competence dilemma}

The 'binary competence' and 'neural plate border' models have provided useful frameworks for understanding cell fate determination during neural, neural crest and placodal segregation (Figure 7A, B). The binary competence model suggests that the ectoderm is subdivided into non-neural/placodal and neural/neural crest domains (Pieper et al., 2012), whilst the neural plate border model suggests cells at the border remain multi-potent and can differentiated into all ectodermal fates (Roellig et al., 2017). Due to the limitations of studying cell fate competence at a population level, transplantation experiments have failed to resolve these two models. 
871 Our co-expression analysis of pan-neural crest and pan-placodal gene modules found that at 872 early stages, most cells at the NPB co-express these modules at similar levels. At later stages, we see a divergence in the level of co-expression between cell populations, with the highest co-expressing cells located at the branching points between the neural crest and placodal lineages (Figure $5 \mathrm{H}$ and $\mathrm{I}$ ). In summary, we find that over time, the putative indeterminate cell population reduces in size until it is restricted to only a small number of bridge cells which lie at the boundary between placodal and neural crest cell clusters at the 4-somite stage. Cell fate allocation within these bridge cells is likely to be driven via transcription factor crossrepression, given the previously characterised cross-repressive interactions taking place between placodal, neural crest and neural specifiers (Brugmann et al., 2004; Christophorou et al., 2009; Schlosser et al., 2008; Wakamatsu et al., 2004).

Given these findings, we propose a 'gradient border' model for cell fate allocation (Figure 7C). This model suggests that cell fate allocation is probabilistic and intimately linked to a cells spatiotemporal positioning. In accordance with the binary competence model, cells located within the lateral NPB will give rise to epidermal and placodal fates, whilst those found medially give rise to neural and neural crest fates. However, instead of a binary segregation of the NPB into neural/neural crest and non-neural/placodal domains, we suggest that a subset of cells at the NPB are kept in an indeterminate state whilst they continue to co-express factors involved in the specification of alternative lineages. Both our co-expression analysis and previous documentation of heterogeneity at the NPB support this model.

892

893

Materials and Methods

895

\section{Data collection and alignment}

897

\section{Single cell dissociation and 10X single-cell mRNA sequencing}

Fertilised chicken eggs were incubated at $38^{\circ} \mathrm{C}$ for $24-32$ hours depending upon the developmental stage collected. Embryos were pinned and had the endoderm and mesoderm removed using dispase $(10 \mathrm{mg} / \mathrm{ml})$ before dissecting the ectodermal region of interest (Figure 1A). Multiple embryos from the same stage were pooled prior to dissociation. Samples were then dissociated in $250 \mu \mathrm{l}$ pre-heated FACSmax cell dissociation solution (Amsbio, T200100) with papain $\left(30 \mathrm{U} / \mathrm{ml}\right.$; Sigma, P3125) for 20 minutes at $37^{\circ} \mathrm{C}$. Samples were gently pipetted 
added to prevent any further dissociation (HBSS; non-acetylated BSA (1 mg/ml; Invitrogen, 10743447); HEPES (0.01M); non-essential amino acids (1x; Thermo Fisher Scientific,

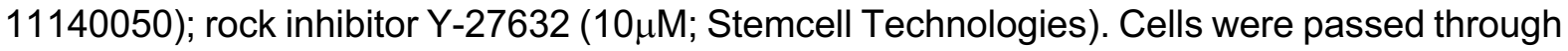
a $20 \mu \mathrm{m}$ cell strainer (Miltenyi Biotech, 130-101-812) to remove any debris, before pelleting and resuspending in $500 \mu \mathrm{l}$ resuspension buffer. To remove any dead or dying cells as well as any remaining doublets, $1 \mu \mathrm{l} 0.1 \mathrm{mg} / \mathrm{ml}$ DAPI was added to the cell suspension before FAC sorting. Given the low number of cells obtained from each embryo, samples for each stage were collected over multiple days and stored in $90 \% \mathrm{MeOH}$ with $10 \%$ resuspension solution after FAC sorting. Prior to sequencing, samples from the same stage were pooled and resuspended in DPBS with $0.5 \%$ non-acetylated BSA and $0.5 \mathrm{U} / \mu \mathrm{l}$ RNAse inhibitor (Roche $3335399001)$.

Cells underwent single-cell mRNA sequencing at the Francis Crick Institute, London. Library preparation was carried out using the 10x 3' single-cell mRNA v3 kit (10x Genomics), before sequencing on the HiSeq 4000 (Illumina) to a target depth of 50k reads/cell. Samples were sequenced in two batches (batch 1: $\mathrm{HH} 4, \mathrm{HH}$, ss4, ss8; batch 2: $\mathrm{HH}$, $\mathrm{HH} 6, \mathrm{HH}$, ss4), with two stages ( $\mathrm{HH} 6$ and ss4) sequenced in both batches to allow for the correction of any potential batch effects. Data from the first batch has previously been used for the characterisation of neural induction (Trevers et al., 2021) (ArrayExpress accession number: E-MTAB-10408).

Read alignment

A custom pipeline was developed in Nextflow (v20.07.1) (Di Tommaso et al., 2017) to handle all GTF processing and read alignment whilst maintaining full reproducibility.

Prior to scRNAseq alignment, the GalGal6 ensembl 97 GTF file was modified to prefix genes from the $Z$ and $W$ sex chromosomes with their respective chromosome IDs (Z- and W-).

933 Furthermore, we noticed several mitochondrial genes in the GTF were missing their chromosome ID, in turn preventing accurately calculating cell percentage mitochondrial content downstream; mitochondrial genes were therefore also prefixed in the GTF (MT-).

936 Finally, we annotated the key neural crest specifier Snai2 in the GTF 937 (ENSGALG00000030902). Reads were processed and aligned to GalGal6 using CellRanger 4.0.0 (10x Genomics). 
940 The aligned reads from CellRanger (BAM) were re-counted using Velocyto (La Manno et al.,

941 2018) to generate spliced and unspliced counts for each gene, which are used in subsequent

942 RNA velocity analysis.

\section{Downstream pre-processing}

All downstream analysis was wrapped into a custom Nextflow pipeline to simplify re-running the analysis by handling parallel processing of data subsets.

Data filtering

Quality control, data filtering, cell clustering, dimensionality reduction and initial data visualisation was carried out using Seurat v4.0.5 (Hao et al., 2021). Data filtering was carried out on each sequencing batch separately before data integration. To ensure the removal of poor-quality cells whilst also minimising over-filtering, we first applied a medium filtering threshold (cells expressing fewer than 1000 genes, more than 6500 genes, or had more than $15 \%$ mitochondrial content were excluded from subsequent analysis) followed by the removal of remaining poor-quality cell clusters.

\section{Dimensionality reduction and clustering} Linear dimensionality reduction was carried out using principal component analysis (PCA). The number of principal components (PCs) used to construct the subsequent shared-nearestneighbour (SNN) graph was determined unbiasedly, using an approach developed by the Harvard Chan Bioinformatics Core (HBC). First, we calculate the PC at which subsequent PCs contribute less than $5 \%$ of standard deviation, and the point where the cumulative variation of PCs exceeds $90 \%$. The larger of these two values is selected. Second, we calculate the PC where the inclusion of consecutive PCs contributes less than $0.1 \%$ additional variation. The smaller value from these two steps is selected as a cut-off.

Louvain clustering was carried out with varying resolutions depending upon the data subset in question. A high clustering resolution (resolution=2) was used to identify poor quality clusters and contaminating cell states. This was to ensure that only poor-quality cells were filtered from the dataset. All other clustering was carried out using a resolution of 0.5. 
975 Cell quality was measured using unique gene and UMI counts. Clusters in which both metrics fell below the 25th percentile relative to the rest of the dataset were considered poor quality and excluded from further analysis.

\section{Data integration}

After the removal of poor-quality cells, cells from the two separate batches were integrated into a single full dataset (Figure 1-figure supplement $1 \mathrm{H}-\mathrm{I}$ ). To avoid over-correction and removal of stage differences, we integrated the data from the two sequencing batches using STACAS v1.1.0 (Andreatta \& Carmona, 2021). This R package allows for alignment of datasets containing partially overlapping cell states.

\section{Regressing out confounding variables}

Before downstream analysis, we regressed out percentage mitochondrial content, cell sex, and cell cycle. After regressing out percentage mitochondrial content, we identified a residual sex effect whereby cells clustered according to the expression of either $\mathrm{W}$ or $\mathrm{Z}$ chromosome genes. This resulted in a duplication of cell clusters, most apparent at $\mathrm{HH} 4$ (Figure 1-figure supplement $1 \mathrm{~A}, \mathrm{~B})$. Cells were k-means clustered according to their expression of $\mathrm{W}$ chromosome genes and classified as either male or female. This classification was used to regress out cell sex. Cell cycle was observed to have a strong effect on cell clustering and was therefore also regressed out during data scaling (Figure 1-figure supplement 1C, D).

\section{Removal of contaminating clusters}

997

We found small contaminating clusters of primordial germ cells, blood islands, mesoderm and endoderm within the dataset (Figure 1-figure supplement 1F, G). To semi-unbiasedly remove these clusters, we calculated the average expression of candidate markers for each of these cell states and removed clusters in which the median expression of any of these modules was greater than the $90^{\text {th }}$ percentile relative to the rest of the dataset (Figure 1-figure supplement 1F", G).

1003

1004

\section{Downstream analysis}

1005

\section{Unbiased cell classification}

1007

Cell states were classified on each stage independently using a binary knowledge matrix 1008 (Supplementary File 1). First, the full dataset was split by stage and then re-scaled and reclustered as described above. Our binary knowledge matrix consists of binarized expression of 76 genes across 24 cell states. For each gene, published in situ hybridisation expression patterns were used to determine expression within each defined cell state. Given that gene 
expression can vary dramatically between stages, and some cell states are known to not be present at specific timepoints (i.e., delaminating neural crest at $\mathrm{HH} 4$ ), we restricted the possible cell states at specific stages. At HH4 there were 7 possible cell states, including nonneural ectoderm (NNE), node, streak, extra-embryonic (EE), early NPB (eNPB), early neural $(\mathrm{eN})$, early caudal neural $(\mathrm{eCN})$. At HH5 there were 17 potential states, this included all cell states in the binary knowledge matrix, except for the later counterparts to the earlier cell states which are ventral forebrain (vFB), forebrain (FB), midbrain (MB), hindbrain (HB), neural crest (NC), delaminating neural crest ( $\mathrm{dNC}$ ) and presumptive epidermis (pEpi). At $\mathrm{HH} 6$ we included the same cell states as $\mathrm{HH} 5$ except for EE and eNPB given that no cell clusters were classified as such at $\mathrm{HH} 5$. For stages $\mathrm{HH} 7$, ss 4 and ss8 we included 17 cell states, excluding the following states which are only found at earlier stages (NNE, node, streak, eN, eCN, eNPB, EE).

For each cell, we calculated the average scaled expression of the genes expressed in each cell state according to our binary knowledge matrix. Cell clusters were then assigned a cell state, depending on which cell state exhibited the highest median expression in that given cell cluster. Assigned cell states at each of the 6 sampled developmental stages were then transferred to the full dataset.

\section{Gene module identification and filtering}

To calculate groups of highly correlated genes, we first filtered genes which do not correlate (Spearman correlation < 0.3) with at least 3 other genes. Then we calculated gene modules using the Antler R package (Delile et al., 2019), which iteratively clusters genes hierarchically based on their gene-gene Spearman correlation. After each iteration, gene modules were filtered to remove gene modules which are not highly expressed (expressed in fewer than 10 cells) or which do not show consistent high expression across genes (fewer than $40 \%$ of genes in the module are expressed after binarization). Gene modules were calculated on different subsets of cells: the full dataset (Figure 3 and Figure 3-figure supplement 1), ss8 (Figure 4 and Figure 5), and the NPB subset (Figure 6). Calculating gene modules on different subsets allowed us to zoom in on specific aspects of ectodermal lineage segregation (for workflow explanation see Figure 3-figure supplement 2).

Many genes correlate across the dataset, but do not show differential expression between cell states and therefore do not provide useful information regarding lineage segregation. Therefore, to identify which gene modules correlate with lineage segregation, modules were filtered to only include those in which more than $50 \%$ of their genes were differentially expressed $(\log F C>0.5$, adjusted $p$-value $<0.001)$ in at least one cell state relative to the rest 
of the dataset. For the NPB subset, this filtering of differential expression was just between the neural crest (NC, dNC) and placodal (aPPR, pPPR, PPR) states rather than between all cell states $(\log F C>0.25$, adjusted $p$-value $<0.001)$.

To remove gene modules which correlate with technical variation, we filtered those in which more than $50 \%$ their genes showed differential expression between sequencing batches $(\log F C>0.25$, adjusted p-value $<0.001)$.

For the gene modules calculated on the full dataset, we were particularly interested in gene modules that segregated into one of the three lineages (neural, neural crest or placodal) at later stages. To focus our analysis on these gene modules, we applied an additional filtering step to only keep gene modules in which more than $50 \%$ of genes were differentially expressed between one of the three lineages at ss8 $(\operatorname{logFC}>0.25$, adjusted $p$-value $<0.001)$. After filtering 9 gene modules remained (Figure 3-figure supplement 2A, Figure 3-Source Data 1). Modules were further manually filtered for visualisation (Figure $3 A$ ).

For the gene modules calculated on the NPB subset we wanted to focus on the co-expression and sequential activation of gene modules that contain well-characterised neural crest and PPR markers. We therefore further filtered NPB gene modules to include only those that had genes that were defined within neural crest, delaminating neural crest or PPR cell states in the binary knowledge matrix. After this final filtering step, 7 gene modules remained (Figure 6E, Figure 6-Source Data 1).

\section{Identification of undetermined cells at the NPB}

To identify which cells expressed competing pan-placodal and pan-neural crest modules at similar levels, we calculated the average expression (normalised counts) of these two gene modules in each cell and then ordered cells by their ratio of pan-placodal to pan-neural crest module expression. Cells were then grouped into 10 evenly sized bins and visualised on UMAPs.

\section{Co-expression distribution plots}

To investigate the distribution of co-expression between cells, co-expression histograms were plotted (Figure 5I). Co-expression values were calculated by multiplying the average normalised expression of the pan-placodal and pan-neural crest gene modules.

\section{Co-expression visualisation}


The average normalised expression of each gene module was scaled between 0 and 1. A $100 \times 100$ two-colour matrix was created using Seurat's BlendMatrix function. Each cell was assigned a colour from this matrix based on their scaled average expression of the gene modules (see Figure $5 \mathrm{H}$ and Figure $6 \mathrm{l}$ legends). Only cells which express both modules above a threshold of 0.3 after scaling were visualised.

\section{RNA velocity}

Spliced and unspliced matrices obtained from Velocyto (La Manno et al., 2018) (see Read Alignment) were analysed using scVelo (Bergen et al., 2020) to generate cell velocity vectors. Velocyto matrices were intersected with each of the data subsets generated in Seurat. First and second order moments were then calculated for each cell across its 20 nearest neighbours using the top 20 PCs. RNA velocity was ran using the dynamical model whilst accounting for differential kinetics; this allows for differential splicing rates between genes as well as differential splicing kinetics between cell states.

Latent time was calculated and used downstream as a measure of a cell's internal clock. When calculating latent time, cells were rooted to the earliest timepoint present in the data subset; in cases where only one stage was present, the cell root was calculated automatically. For all latent time estimates, we used a diffusion weighting of 0.2 .

\section{Modelling gene expression dynamics}

Lineage inference was calculated using CellRank, which uses a probabilistic approach to assign cell lineage (Lange et al., 2022). Cell transition matrices were calculated using RNA velocity ( 0.8 weighting) and nearest neighbour graph ( 0.2 weighting) kernels. A softmax scale of 4 was set to prevent transitions tending towards orthogonality. When predicting cell transitions, terminal states were specified according to the lineages which we were wanting to model gene dynamics across. For the full dataset, we predicted cell transitions towards either neural, placodal or neural crest lineages. Terminal states were specified as: delaminating neural crest and neural crest cell states at ss 4 and ss 8 for the neural crest lineage; hindbrain, midbrain, and forebrain cell states at ss4 and ss8 for the neural lineage; anterior PPR, posterior PPR and PPR cell states at ss4 and ss8 for the placodal lineage (Figure 3-figure supplement 1C). For our NPB subset we predicted cell transitions towards either placodal or neural crest lineages, with terminal states specified as: delaminating neural crest and neural crest cell states at ss8 for the neural crest lineage; aPPR and PPR cell states at ss8 for the placodal lineage. 
1121 Gene expression dynamics were calculated by fitting general additive models (GAM), which 1122 modelled scaled gene expression as a function of latent time using the mgcv package in $\mathrm{R}$. 1123 Lineage absorption probability obtained from CellRank were used to weight a GAM for each

1124 of the predicted lineages. To generate a smoothed prediction of gene expression change, a 1125 cubic spline was fitted with knots=4. When modelling the dynamics of entire gene modules, 1126 all genes within a given module were included in the GAM.

\section{Data availability and reproducibility}

10x single cell RNAseq was carried out in two batches and is available under two separate accession numbers (ArrayExpress: E-MTAB-10408 and E-MTAB-1144). Our NGS alignments and downstream analysis have been wrapped into custom Nextflow pipelines allowing for full reproducibility. For the code used in this analysis, including links to our Docker containers, see our GitHub repository at https://github.com/alexthiery/10x_neural_plate_border.

\section{Acknowledgements}

We thank Teresa Rayon for assistance with establishing our single cell dissociation protocol and the Advanced Sequencing Facility at the Francis Crick Institute for the single cell RNA sequencing. We would also like to thank Igor Adameyko and Artem Artemov for guidance on co-expression analysis, Chantal Hubens for technical support, Alessandra Vigilante for comments on the manuscript, and Streit and Luscombe lab groups for helpful discussions. This work was supported by the Wellcome Trust $(108874 / \mathrm{B} / 15 / \mathrm{Z})$ and the BBSRC (BB/S005536/1; BB/R006342/1).

\section{Author contributions}

A.T., A.S., J.B. designed study. A.T., A.B., E.H., A.S. collected samples and processed for scRNAseq. A.T., C.C. developed analysis pipeline and carried out data alignment. A.T., E.H. carried out downstream computational analysis of scRNAseq data. A.T., A.B., E.H., A.S. made figures and wrote manuscript. All authors provided feedback on the manuscript. 


\section{References}

Adams, M. S., Gammill, L. S., \& Bronner-Fraser, M. (2008). Discovery of transcription factors and other candidate regulators of neural crest development. Dev Dyn, 237(4), 1021-1033. doi:10.1002/dvdy. 21513

Ahrens, K., \& Schlosser, G. (2005). Tissues and signals involved in the induction of placodal Six1 expression in Xenopus laevis. Dev Biol, 288(1), 40-59.

Andreatta, M., \& Carmona, S. J. (2021). STACAS: Sub-Type Anchor Correction for Alignment in Seurat to integrate single-cell RNA-seq data. Bioinformatics, 37(6), 882-884. doi:10.1093/bioinformatics/btaa755

Baker, C. V., \& Bronner-Fraser, M. (1997). The origins of the neural crest. Part I: embryonic induction. Mech Dev, 69(1-2), 3-11. doi:10.1016/s0925-4773(97)00132-9

Baker, C. V., \& Bronner-Fraser, M. (2001). Vertebrate cranial placodes I. Embryonic induction. Dev Biol, 232(1), 1-61. doi:10.1006/dbio.2001.0156

Barembaum, M., \& Bronner-Fraser, M. (2007). Spalt4 mediates invagination and otic placode gene expression in cranial ectoderm. Development, 134(21), 3805-3814. doi:10.1242/dev.02885

Barth, K. A., Kishimoto, Y., Rohr, K. B., Seydler, C., Schulte-Merker, S., \& Wilson, S. W. (1999). Bmp activity establishes a gradient of positional information throughout the entire neural plate. Development, 126(22), 4977-4987.

Basch, M. L., Bronner-Fraser, M., \& Garcia-Castro, M. I. (2006). Specification of the neural crest occurs during gastrulation and requires Pax7. Nature, 441(7090), 218-222.

Bell, G. W., Yatskievych, T. A., \& Antin, P. B. (2004). GEISHA, a whole-mount in situ hybridization gene expression screen in chicken embryos. Dev Dyn, 229(3), 677-687. doi:10.1002/dvdy.10503

Bergen, V., Lange, M., Peidli, S., Wolf, F. A., \& Theis, F. J. (2020). Generalizing RNA velocity to transient cell states through dynamical modeling. Nat Biotechnol, 38(12), 1408-1414. doi:10.1038/s41587-020-0591-3

Betancur, P., Bronner-Fraser, M., \& Sauka-Spengler, T. (2010). Assembling neural crest regulatory circuits into a gene regulatory network. Annu Rev Cell Dev Biol, 26, 581-603. doi:10.1146/annurev.cellbio.042308.113245

Bhat, N., Kwon, H. J., \& Riley, B. B. (2013). A gene network that coordinates preplacodal competence and neural crest specification in zebrafish. Dev Biol, 373(1), 107-117. doi:10.1016/j.ydbio.2012.10.012

Bhattacharyya, S., Bailey, A. P., Bronner-Fraser, M., \& Streit, A. (2004). Segregation of lens and olfactory precursors from a common territory: cell sorting and reciprocity of Dlx5 and Pax6 expression. Dev Biol, 271(2), 403-414. doi:10.1016/j.ydbio.2004.04.010

Brugmann, S. A., Pandur, P. D., Kenyon, K. L., Pignoni, F., \& Moody, S. A. (2004). Six1 promotes a placodal fate within the lateral neurogenic ectoderm by functioning as both a transcriptional activator and repressor. Development, 131(23), 5871-5881.

Buitrago-Delgado, E., Nordin, K., Rao, A., Geary, L., \& LaBonne, C. (2015). Shared regulatory programs suggest retention of blastula-stage potential in neural crest cells. Science, 348(6241), 13321335. doi:10.1126/science.aaa3655

Chapman, S. C., Schubert, F. R., Schoenwolf, G. C., \& Lumsden, A. (2002). Analysis of spatial and temporal gene expression patterns in blastula and gastrula stage chick embryos. Dev Biol, 245(1), 187-199. doi:10.1006/dbio.2002.0641

Chen, B., Kim, E.-H., \& Xu, P.-X. (2009). Initiation of olfactory placode development and neurogenesis is blocked in mice lacking both Six1 and Six4. Developmental Biology, 326(1), 75-85.

Christophorou, N. A., Bailey, A. P., Hanson, S., \& Streit, A. (2009). Activation of Six1 target genes is required for sensory placode formation. Dev Biol, 336(2), 327-336.

Delile, J., Rayon, T., Melchionda, M., Edwards, A., Briscoe, J., \& Sagner, A. (2019). Single cell transcriptomics reveals spatial and temporal dynamics of gene expression in the developing mouse spinal cord. Development, 146(12). doi:10.1242/dev.173807 
Di Tommaso, P., Chatzou, M., Floden, E. W., Barja, P. P., Palumbo, E., \& Notredame, C. (2017). Nextflow enables reproducible computational workflows. Nat Biotechnol, 35(4), 316-319. doi:10.1038/nbt.3820

Ezin, A. M., Fraser, S. E., \& Bronner-Fraser, M. (2009). Fate map and morphogenesis of presumptive neural crest and dorsal neural tube. Dev Biol, 330(2), 221-236. doi:10.1016/j.ydbio.2009.03.018

Garcia-Castro, M. I., Marcelle, C., \& Bronner-Fraser, M. (2002). Ectodermal Wnt function as a neural crest inducer. Science, 297(5582), 848-851.

Grocott, T., Tambalo, M., \& Streit, A. (2012). The peripheral sensory nervous system in the vertebrate head: a gene regulatory perspective. Dev Biol, 370(1), 3-23. doi:10.1016/j.ydbio.2012.06.028

Hao, Y., Hao, S., Andersen-Nissen, E., Mauck, W. M., III., Zheng, S., Butler, A., Lee, M. J., Wilk, A. J., Darby, C., Zager, M., Hoffman, P., Stoeckius, M., Papalexi, E., Mimitou, E. P., Jain, J., Srivastava, A., Stuart, T., Fleming, L. M., Yeung, B., Rogers, A. J., McElrath, J. M., Blish, C. A., Gottardo, R., Smibert, P., \& Satija, R. (2021). Integrated analysis of multimodal single-cell data. Cell, 184(13), 3573-3587 e3529. doi:10.1016/j.cell.2021.04.048

Heeg-Truesdell, E., \& LaBonne, C. (2006). Neural induction in Xenopus requires inhibition of Wnt-betacatenin signaling. Developmental Biology, 298(1), 71-86.

Hemmati-Brivanlou, A., Kelly, O. G., \& Melton, D. A. (1994). Follistatin, an antagonist of activin, is expressed in the Spemann organizer and displays direct neuralizing activity. Cell, 77(2), 283295.

Hidalgo-Sanchez, M., Millet, S., Bloch-Gallego, E., \& Alvarado-Mallart, R. M. (2005). Specification of the meso-isthmo-cerebellar region: the Otx2/Gbx2 boundary. Brain Res Brain Res Rev, 49(2), 134-149. doi:10.1016/j.brainresrev.2005.01.010

Hidalgo-Sanchez, M., Millet, S., Simeone, A., \& Alvarado-Mallart, R. M. (1999). Comparative analysis of Otx2, Gbx2, Pax2, Fgf8 and Wnt1 gene expressions during the formation of the chick midbrain/hindbrain domain. Mech Dev, 81(1-2), 175-178. doi:10.1016/s0925-4773(98)00224-x

Hintze, M., Prajapati, R. S., Tambalo, M., Christophorou, N. A. D., Anwar, M., Grocott, T., \& Streit, A. (2017). Cell interactions, signals and transcriptional hierarchy governing placode progenitor induction. Development, 144(15), 2810-2823. doi:10.1242/dev.147942

Hollyday, M., McMahon, J. A., \& McMahon, A. P. (1995). Wnt expression patterns in chick embryo nervous system. Mech Dev, 52(1), 9-25. doi:10.1016/0925-4773(95)00385-e

Hong, C. S., \& Saint-Jeannet, J. P. (2007). The activity of Pax3 and Zic1 regulates three distinct cell fates at the neural plate border. Mol Biol Cell, 18(6), 2192-2202. doi:10.1091/mbc.e06-11-1047

Kwon, H. J., Bhat, N., Sweet, E. M., Cornell, R. A., \& Riley, B. B. (2010). Identification of early requirements for preplacodal ectoderm and sensory organ development. PLoS Genet, 6(9). doi:10.1371/journal.pgen.1001133

La Manno, G., Soldatov, R., Zeisel, A., Braun, E., Hochgerner, H., Petukhov, V., Lidschreiber, K., Kastriti, M. E., Lonnerberg, P., Furlan, A., Fan, J., Borm, L. E., Liu, Z., van Bruggen, D., Guo, J., He, X., Barker, R., Sundstrom, E., Castelo-Branco, G., Cramer, P., Adameyko, I., Linnarsson, S., \& Kharchenko, P. V. (2018). RNA velocity of single cells. Nature, 560(7719), 494-498. doi:10.1038/s41586-018-0414-6

Lange, M., Bergen, V., Klein, M., Setty, M., Reuter, B., Bakhti, M., Lickert, H., Ansari, M., Schniering, J., Schiller, H. B., Pe'er, D., \& Theis, F. J. (2022). CellRank for directed single-cell fate mapping. Nat Methods. doi:10.1038/s41592-021-01346-6

Liberatore, C. M., Searcy-Schrick, R. D., \& Yutzey, K. E. (2000). Ventricular expression of tbx5 inhibits normal heart chamber development. Dev Biol, 223(1), 169-180. doi:10.1006/dbio.2000.9748

Liem, K. F., Jr., Tremml, G., Roelink, H., \& Jessell, T. M. (1995). Dorsal differentiation of neural plate cells induced by BMP-mediated signals from epidermal ectoderm. Cell, 82(6), 969-979.

Linker, C., \& Stern, C. D. (2004). Neural induction requires BMP inhibition only as a late step, and involves signals other than FGF and Wnt antagonists. Development, 131(22), 5671-5681.

Litsiou, A., Hanson, S., \& Streit, A. (2005). A balance of FGF, Wnt and BMP signalling positions the future placode territory in the head. Development, 132, 4051-4062. 
Londin, E. R., Niemiec, J., \& Sirotkin, H. I. (2005). Chordin, FGF signalling, and mesodermal factors cooperate in zebrafish neural induction. Dev Biol, 279(1), 1-19.

Marchant, L., Linker, C., Ruiz, P., Guerrero, N., \& Mayor, R. (1998). The inductive properties of mesoderm suggest that the neural crest cells are specified by a BMP gradient. Dev Biol, 198(2), 319-329.

McLarren, K. W., Litsiou, A., \& Streit, A. (2003). DLX5 positions the neural crest and preplacode region at the border of the neural plate. Dev Biol, 259(1), 34-47. doi:10.1016/s0012-1606(03)00177-5

Mehdizadeh, T., Majumdar, H. D., Ahsan, S., Tavares, A. L. P., \& Moody, S. A. (2021). Mutations in SIX1 Associated with Branchio-oto-Renal Syndrome (BOR) Differentially Affect Otic Expression of Putative Target Genes. J Dev Biol, 9(3). doi:10.3390/jdb9030025

Milet, C., Maczkowiak, F., Roche, D. D., \& Monsoro-Burq, A. H. (2013). Pax3 and Zic1 drive induction and differentiation of multipotent, migratory, and functional neural crest in Xenopus embryos. Proc Natl Acad Sci U S A, 110(14), 5528-5533. doi:10.1073/pnas.1219124110

Monsoro-Burq, A. H. (2015). PAX transcription factors in neural crest development. Semin Cell Dev Biol, 44, 87-96. doi:10.1016/j.semcdb.2015.09.015

Monsoro-Burq, A. H., Fletcher, R. B., \& Harland, R. M. (2003). Neural crest induction by paraxial mesoderm in Xenopus embryos requires FGF signals. Development, 130(14), 3111-3124.

Ohuchi, H., Tomonari, S., Itoh, H., Mikawa, T., \& Noji, S. (1999). Identification of chick rax/rx genes with overlapping patterns of expression during early eye and brain development. Mech Dev, 85(12), 193-195. doi:10.1016/s0925-4773(99)00094-5

Ota, K., Nagai, H., \& Sheng, G. (2007). Expression and hypoxic regulation of hif1alpha and hif2alpha during early blood and endothelial cell differentiation in chick. Gene Expr Patterns, 7(7), 761766. doi:10.1016/j.modgep.2007.05.007

Ozaki, H., Nakamura, K., Funahashi, J., Ikeda, K., Yamada, G., Tokano, H., Okamura, H. O., Kitamura, K., Muto, S., Kotaki, H., Sudo, K., Horai, R., Iwakura, Y., \& Kawakami, K. (2004). Six1 controls patterning of the mouse otic vesicle. Development, 131(3), 551-562.

Patthey, C., Gunhaga, L., \& Edlund, T. (2008). Early development of the central and peripheral nervous systems is coordinated by Wht and BMP signals. PLOS ONE, 3(2), e1625. doi:10.1371/journal.pone.0001625

Patthey, C., Schlosser, G., \& Shimeld, S. M. (2014). The evolutionary history of vertebrate cranial placodes--l: cell type evolution. Dev Biol, 389(1), 82-97. doi:10.1016/j.ydbio.2014.01.017

Paxton, C. N., Bleyl, S. B., Chapman, S. C., \& Schoenwolf, G. C. (2010). Identification of differentially expressed genes in early inner ear development. Gene Expr Patterns, 10(1), 31-43. doi:10.1016/j.gep.2009.11.002

Pera, E., Stein, S., \& Kessel, M. (1999). Ectodermal patterning in the avian embryo: epidermis versus neural plate. Development, 126(1), 63-73.

Pieper, M., Ahrens, K., Rink, E., Peter, A., \& Schlosser, G. (2012). Differential distribution of competence for panplacodal and neural crest induction to non-neural and neural ectoderm. Development, 139(6), 1175-1187. doi:10.1242/dev.074468

Pla, P., \& Monsoro-Burq, A. H. (2018). The neural border: Induction, specification and maturation of the territory that generates neural crest cells. Dev Biol, 444 Suppl 1, S36-S46. doi:10.1016/j.ydbio.2018.05.018

Plouhinec, J. L., Roche, D. D., Pegoraro, C., Figueiredo, A. L., Maczkowiak, F., Brunet, L. J., Milet, C., Vert, J. P., Pollet, N., Harland, R. M., \& Monsoro-Burq, A. H. (2014). Pax3 and Zic1 trigger the early neural crest gene regulatory network by the direct activation of multiple key neural crest specifiers. Dev Biol, 386(2), 461-472. doi:10.1016/j.ydbio.2013.12.010

Prasad, M. S., Charney, R. M., \& Garcia-Castro, M. I. (2019). Specification and formation of the neural crest: Perspectives on lineage segregation. Genesis, 57(1), e23276. doi:10.1002/dvg.23276

Prasad, M. S., Sauka-Spengler, T., \& LaBonne, C. (2012). Induction of the neural crest state: control of stem cell attributes by gene regulatory, post-transcriptional and epigenetic interactions. Dev Biol, 366(1), 10-21. doi:10.1016/j.ydbio.2012.03.014 
bioRxiv preprint doi: https://doi.org/10.1101/2022.02.15.480567; this version posted February $16,2022$. The copyright holder for this preprint (which was not certified by peer review) is the author/funder, who has granted bioRxiv a license to display the preprint in perpetuity. It is made available under aCC-BY-NC-ND 4.0 International license.

Reichert, S., Randall, R. A., \& Hill, C. S. (2013). A BMP regulatory network controls ectodermal cell fate decisions at the neural plate border. Development, 140(21), 4435-4444. doi:10.1242/dev.098707

Rex, M., Orme, A., Uwanogho, D., Tointon, K., Wigmore, P. M., Sharpe, P. T., \& Scotting, P. J. (1997). Dynamic expression of chicken Sox2 and Sox3 genes in ectoderm induced to form neural tissue. Dev Dyn, 209(3), 323-332. doi:10.1002/(SICl)1097-0177(199707)209:3<323::AIDAJA7>3.0.CO;2-K

Roellig, D., Tan-Cabugao, J., Esaian, S., \& Bronner, M. E. (2017). Dynamic transcriptional signature and cell fate analysis reveals plasticity of individual neural plate border cells. Elife, 6. doi:10.7554/eLife. 21620

Rothstein, M., \& Simoes-Costa, M. (2020). Heterodimerization of TFAP2 pioneer factors drives epigenomic remodeling during neural crest specification. Genome Res, 30(1), 35-48. doi:10.1101/gr.249680.119

Sasai, Y., Lu, B., Steinbeisser, H., \& De Robertis, E. M. (1995). Regulation of neural induction by the Chd and Bmp-4 antagonistic patterning signals in Xenopus. Nature, 376(6538), 333-336.

Schlosser, G. (2006). Induction and specification of cranial placodes. Dev Biol, 294(2), 303-351. doi:10.1016/j.ydbio.2006.03.009

Schlosser, G. (2014). Early embryonic specification of vertebrate cranial placodes. Wiley Interdiscip Rev Dev Biol, 3(5), 349-363. doi:10.1002/wdev.142

Schlosser, G., Awtry, T., Brugmann, S. A., Jensen, E. D., Neilson, K., Ruan, G., Stammler, A., Voelker, D., Yan, B., Zhang, C., Klymkowsky, M. W., \& Moody, S. A. (2008). Eya1 and Six1 promote neurogenesis in the cranial placodes in a SoxB1-dependent fashion. Dev Biol, 320(1), 199-214. doi:10.1016/j.ydbio.2008.05.523

Sheng, G., \& Stern, C. D. (1999). Gata2 and Gata3: novel markers for early embryonic polarity and for non-neural ectoderm in the chick embryo. Mech Dev, 87(1-2), 213-216. doi:10.1016/s09254773(99)00150-1

Simoes-Costa, M., \& Bronner, M. E. (2015). Establishing neural crest identity: a gene regulatory recipe. Development, 142(2), 242-257. doi:10.1242/dev.105445

Soldatov, R., Kaucka, M., Kastriti, M. E., Petersen, J., Chontorotzea, T., Englmaier, L., Akkuratova, N., Yang, Y., Haring, M., Dyachuk, V., Bock, C., Farlik, M., Piacentino, M. L., Boismoreau, F., Hilscher, M. M., Yokota, C., Qian, X., Nilsson, M., Bronner, M. E., Croci, L., Hsiao, W. Y., Guertin, D. A., Brunet, J. F., Consalez, G. G., Ernfors, P., Fried, K., Kharchenko, P. V., \& Adameyko, I. (2019). Spatiotemporal structure of cell fate decisions in murine neural crest. Science, 364(6444). doi:10.1126/science.aas9536

Steventon, B., Araya, C., Linker, C., Kuriyama, S., \& Mayor, R. (2009). Differential requirements of BMP and Wnt signalling during gastrulation and neurulation define two steps in neural crest induction. Development, 136(5), 771-779. doi:10.1242/dev.029017

Steventon, B., Mayor, R., \& Streit, A. (2012). Mutual repression between Gbx2 and Otx2 in sensory placodes reveals a general mechanism for ectodermal patterning. Dev Biol, 367(1), 55-65. doi:10.1016/j.ydbio.2012.04.025

Streit, A. (2002). Extensive cell movements accompany formation of the otic placode. Dev Biol, 249(2), 237-254. doi:10.1006/dbio.2002.0739

Streit, A., Berliner, A. J., Papanayotou, C., Sirulnik, A., \& Stern, C. D. (2000). Initiation of neural induction by FGF signalling before gastrulation. Nature, 406(6791), 74-78.

Streit, A., Lee, K. J., Woo, I., Roberts, C., Jessell, T. M., \& Stern, C. D. (1998). Chordin regulates primitive streak development and the stability of induced neural cells, but is not sufficient for neural induction in the chick embryo. Development, 125(3), 507-519.

Streit, A., \& Stern, C. D. (1999). Establishment and maintenance of the border of the neural plate in the chick: involvement of FGF and BMP activity. Mech Dev, 82(1-2), 51-66.

Stuhlmiller, T. J., \& García-Castro, M. I. (2012). FGF/MAPK signaling is required in the gastrula epiblast for avian neural crest induction. Development, 139(2), 289-300. doi:10.1242/dev.070276 
Stundl, J., Bertucci, P. Y., Lauri, A., Arendt, D., \& Bronner, M. E. (2021). Evolution of new cell types at the lateral neural border. Curr Top Dev Biol, 141, 173-205. doi:10.1016/bs.ctdb.2020.11.005

Thiery, A., Buzzi, A. L., \& Streit, A. (2020). Cell fate decisions during the development of the peripheral nervous system in the vertebrate head. Curr Top Dev Biol, 139, 127-167. doi:10.1016/bs.ctdb.2020.04.002

Trevers, K. E., Lu, H. C., Yang, Y., Thiery, A., Strobl, A. C., Pálinkášová, B., de Oliveira, N. M. M., de Almeida, I. M., Khan, M. A. F., Moncaut, N., Luscombe, N. M., Dale, L., Streit, A., \& Stern, C. D. (2021). A gene regulatory network for neural induction. bioRxiv, 2021.2004.2016.440164. doi:10.1101/2021.04.16.440164

Trevers, K. E., Prajapati, R. S., Hintze, M., Stower, M. J., Strobl, A. C., Tambalo, M., Ranganathan, R., Moncaut, N., Khan, M. A. F., Stern, C. D., \& Streit, A. (2018). Neural induction by the node and placode induction by head mesoderm share an initial state resembling neural plate border and ES cells. Proc Natl Acad Sci U S A, 115(2), 355-360. doi:10.1073/pnas.1719674115

Wakamatsu, Y., Endo, Y., Osumi, N., \& Weston, J. A. (2004). Multiple roles of Sox2, an HMG-box transcription factor in avian neural crest development. Dev Dyn, 229(1), 74-86. doi:10.1002/dvdy.10498

Williams, R. M., Lukoseviciute, M., Sauka-Spengler, T., \& Bronner, M. E. (2022). Single-cell atlas of early chick development reveals gradual segregation of neural crest lineage from the neural plate border during neurulation. Elife, 11. doi:10.7554/eLife.74464

Wilson, S. I., Graziano, E., Harland, R., Jessell, T. M., \& Edlund, T. (2000). An early requirement for FGF signalling in the acquisition of neural cell fate in the chick embryo. Curr Biol, 10(8), 421429.

Yardley, N., \& Garcia-Castro, M. I. (2012). FGF signaling transforms non-neural ectoderm into neural crest. Dev Biol, 372(2), 166-177. doi:10.1016/j.ydbio.2012.09.006 

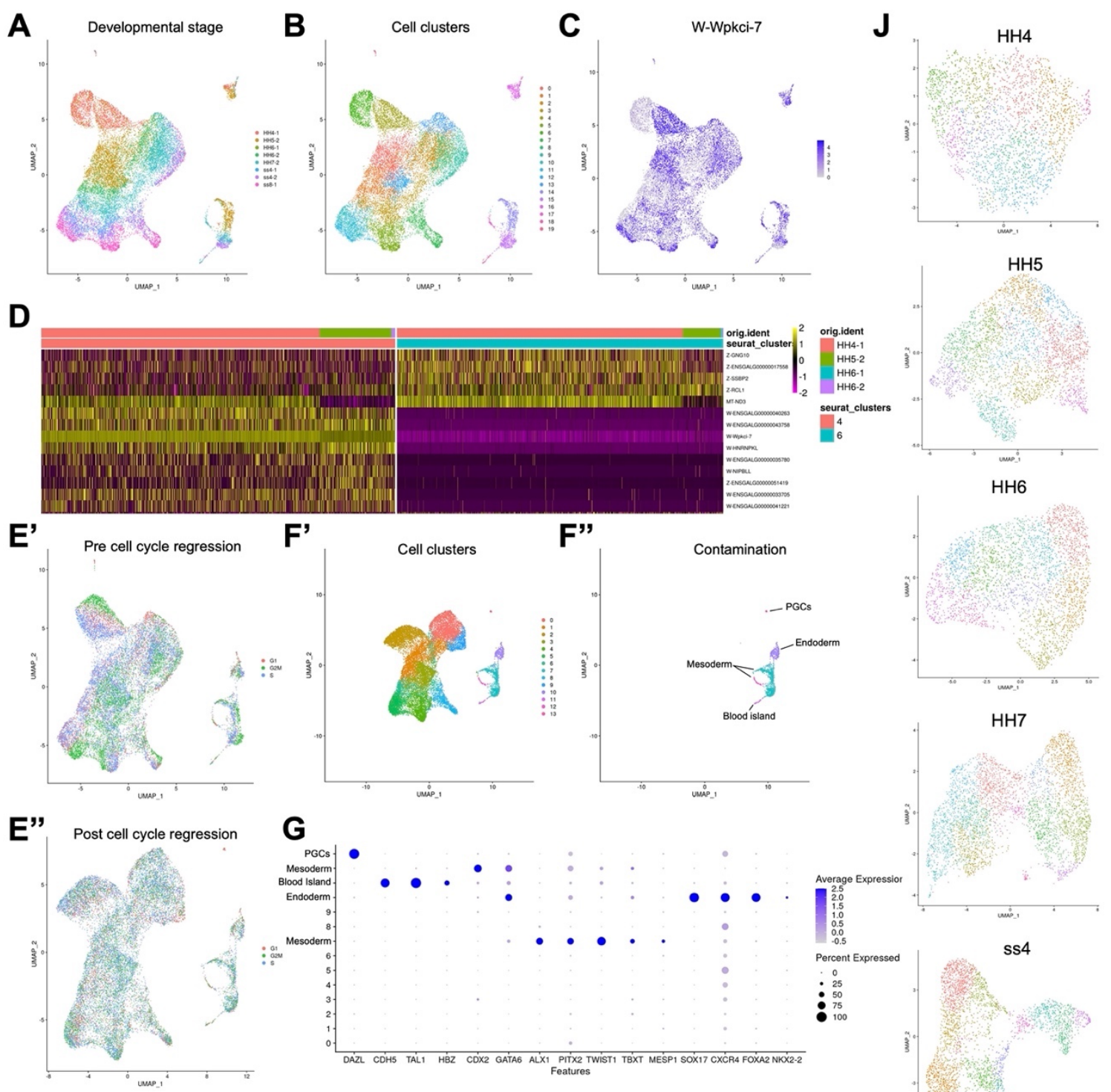

$\mathbf{H}$
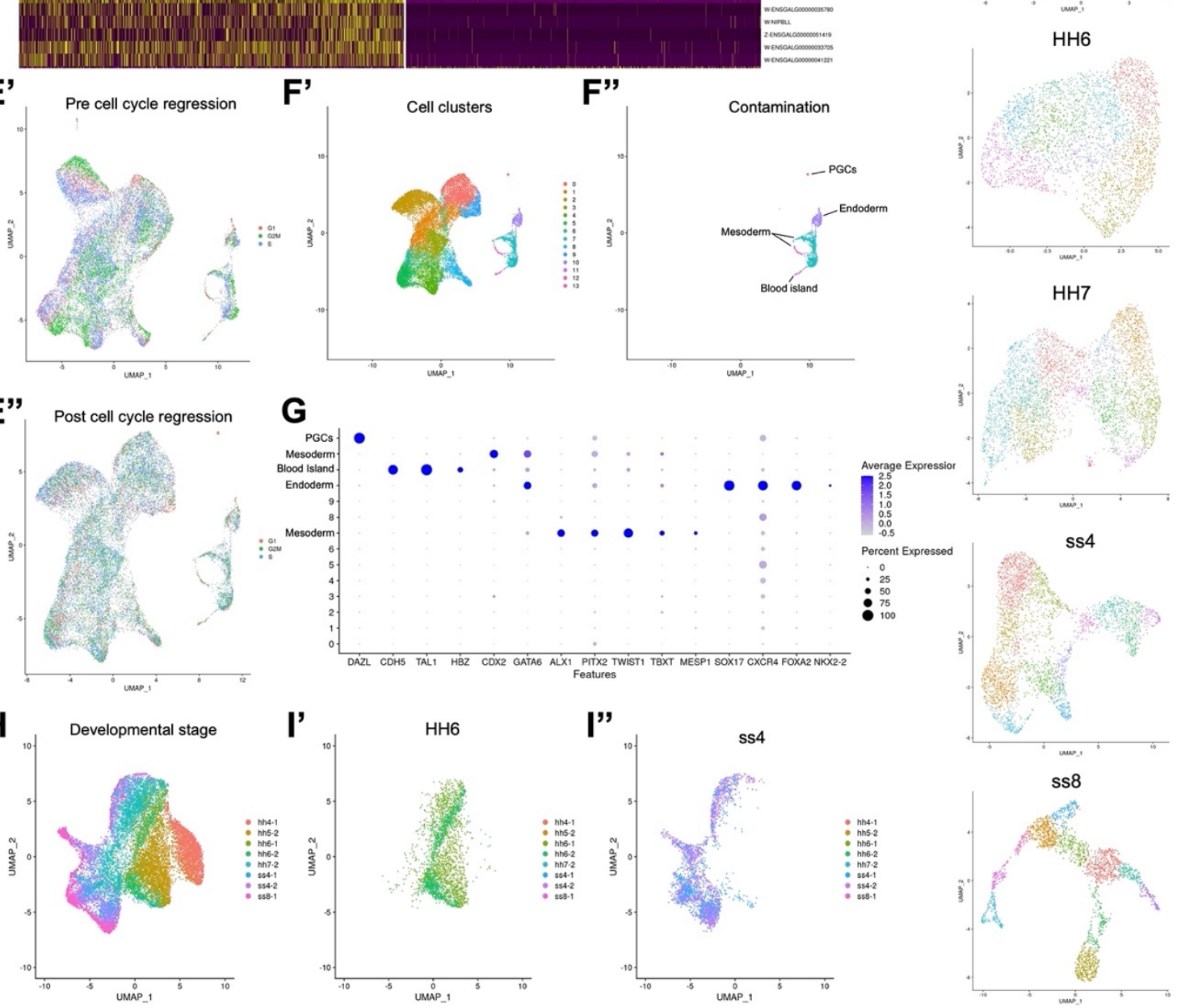

$\mathrm{HH} 7$

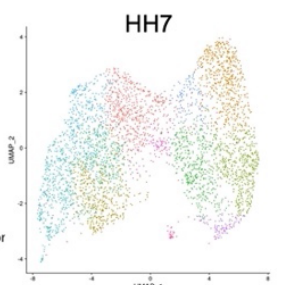

ss4

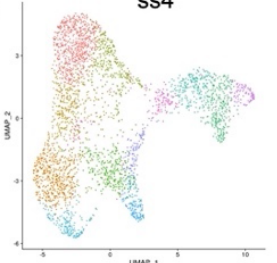

Figure 1-figure supplement 1. (A-B) UMAP of full dataset prior to regressing out confounding factors. Cells are coloured by developmental stage (A) and Seurat cell clusters (B). (C) Feature plot displaying W (male) sex-chromosome gene Wpkci-7, illustrating the cell sex effect after clustering. (D) Heatmap of selected sex genes across clusters 4 and 6 . These clusters contain mostly cells from $\mathrm{HH} 4$ and highlight the clear sex effect of $\mathrm{W}$ and $\mathrm{Z}$ chromosome genes between these clusters. (E) UMAP of cells prior to (E') and post (E') regressing out the cell cycle effect. (F') UMAP displaying cell clusters after regressing out sex and cell cycle effects. (F') UMAP highlighting contaminating cell states following cell state classification. These include primordial germ cells (PGCs), mesoderm, endoderm, and blood islands. (G) 
bioRxiv preprint doi: https://doi.org/10.1101/2022.02.15.480567; this version posted February 16, 2022. The copyright holder for this

preprint (which was not certified by peer review) is the author/funder, who has granted bioRxiv a license to display the preprint in perpetuity. It is made available under aCC-BY-NC-ND 4.0 International license.

1393 Dotplot for the expression of genes used for identification of contaminating cell clusters. $(\mathbf{H})$ 1394 UMAP of all cells after filtering of contaminating populations and regressing out confounding 1395 variables. Cells are coloured by developmental stage. (I) UMAP plots showing successful integration of two sequencing batches at stages HH6 (I') and ss4 (I'). (J) UMAPs displaying 1397 cell clusters calculated at each developmental timepoint. Individual stages are subset from the 1398 full filtered dataset displayed in $(H)$.

1399

1400 
A
I

$A D M P$

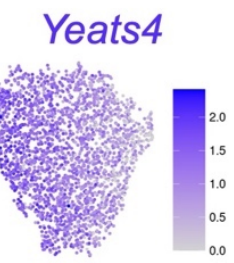

Tfap2a

DIx5

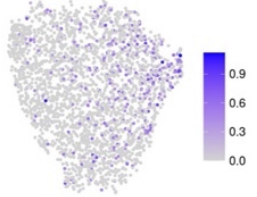

B
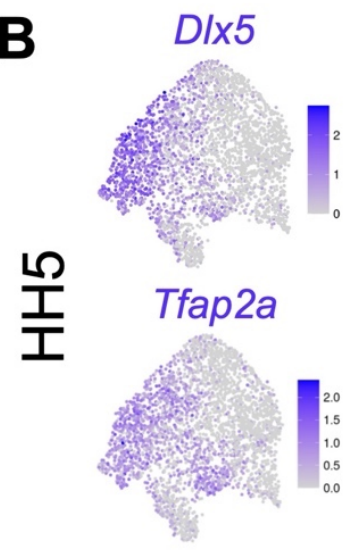

C Tfap2a

Tfap2c

丢
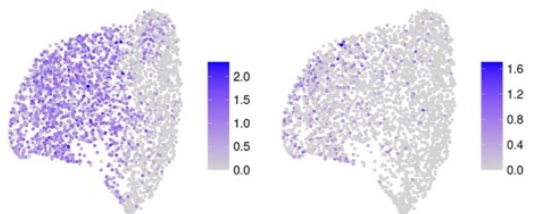

Six1

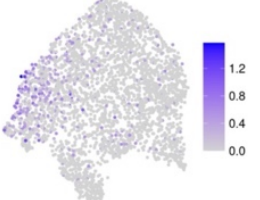

Tfap2c

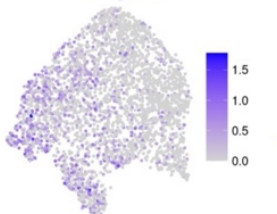

Msx1
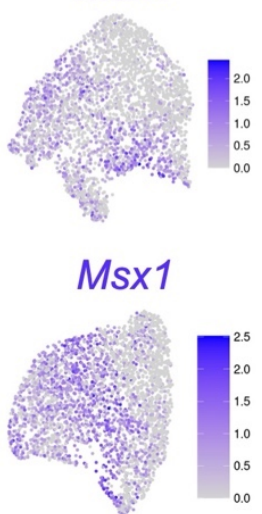

Six3
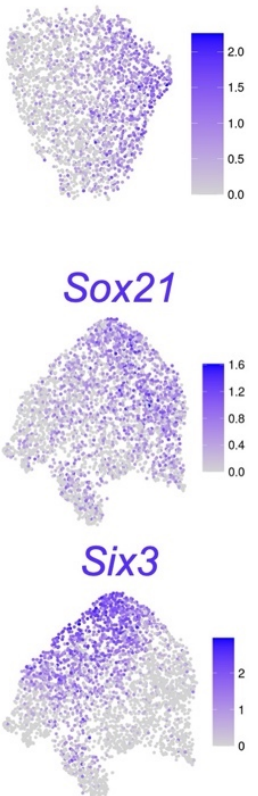

Sox21

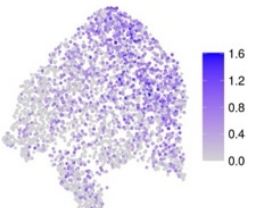

Six3

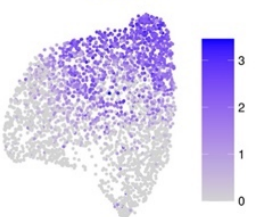

Bmp4

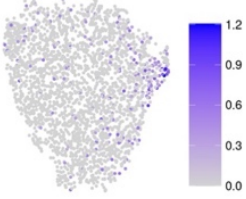

Yeats4

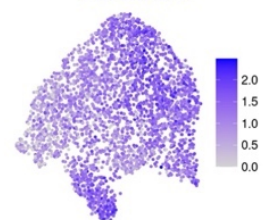

Gbx2

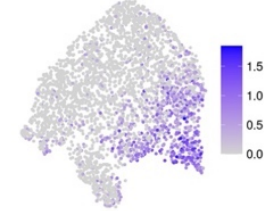

Gbx2

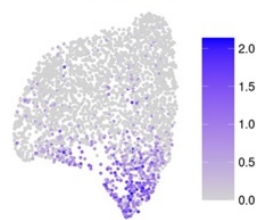

Figure 1-figure supplement 2. Feature plots showing expression of marker genes on UMAPs of developmental stages $\mathrm{HH} 4$ (A), $\mathrm{HH} 5$ (B) and $\mathrm{HH} 6$ (C). 


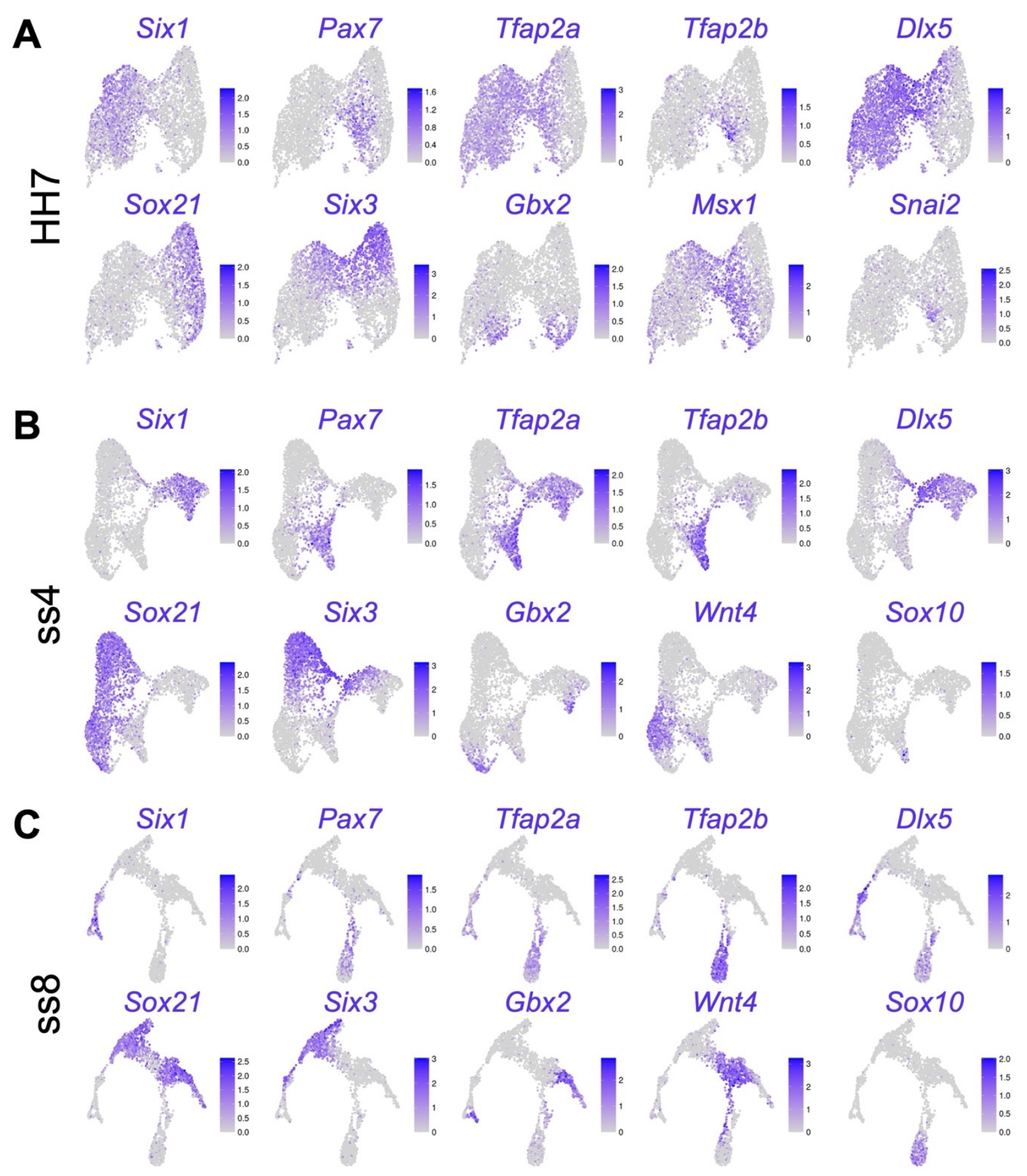

Figure 2-figure supplement 1. Feature plots showing expression of marker genes on UMAPs of developmental stages $\mathrm{HH} 7$ (A), ss4 (B) and ss8 (C). 


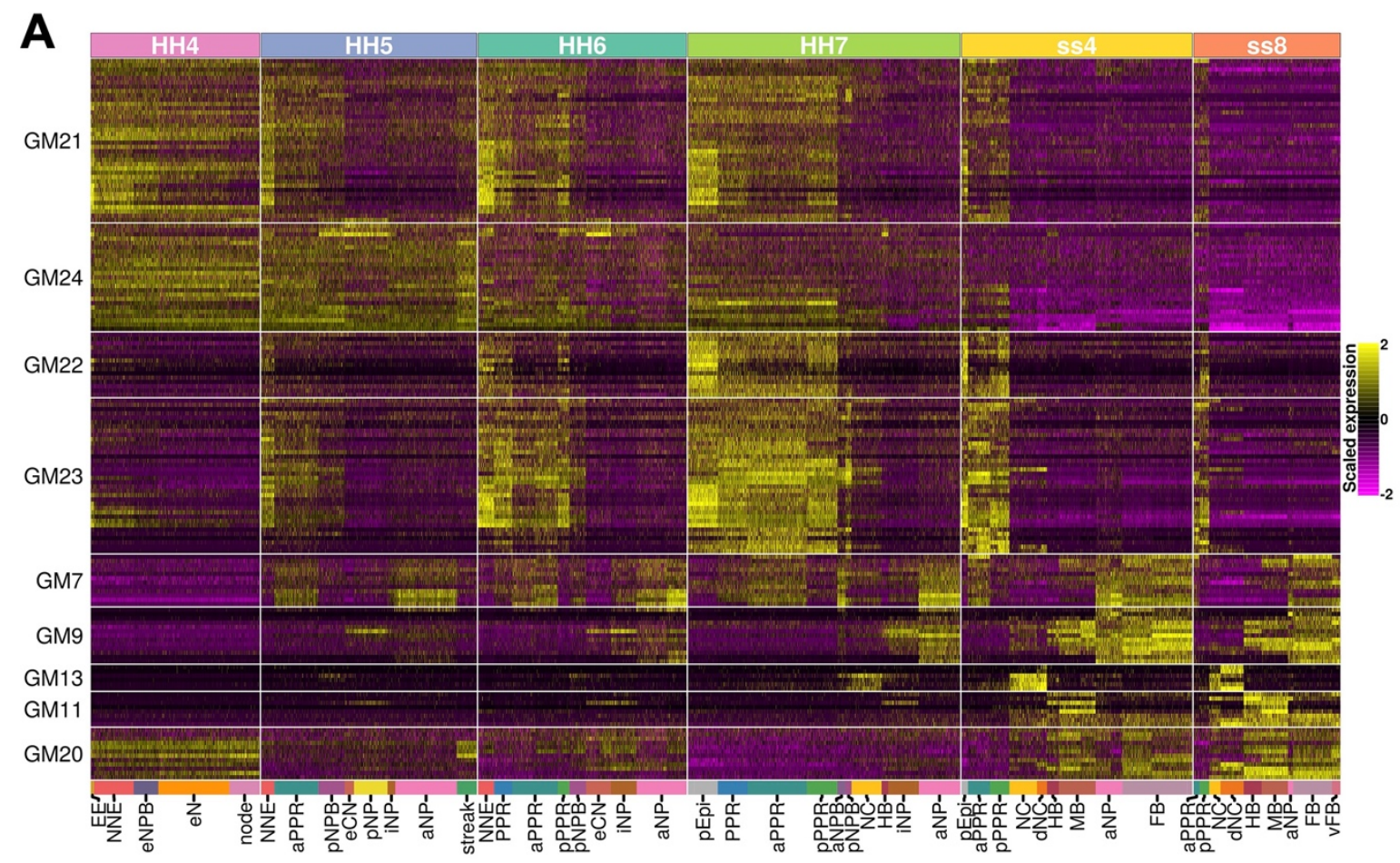

\section{B}

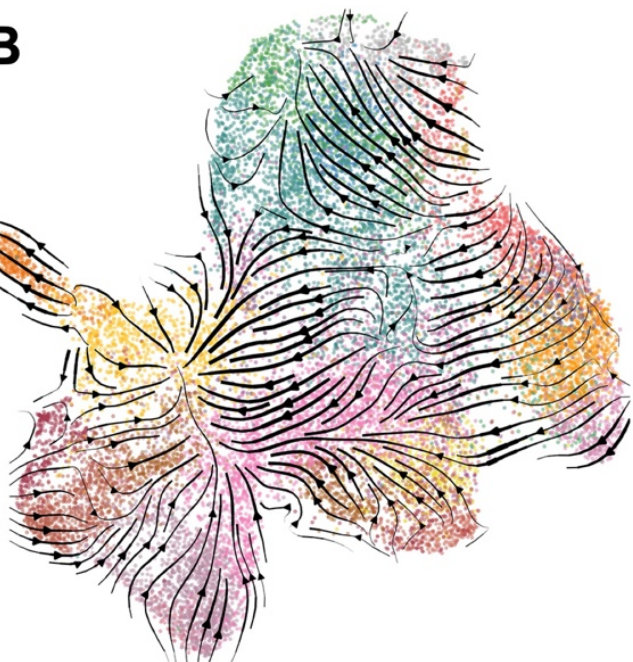

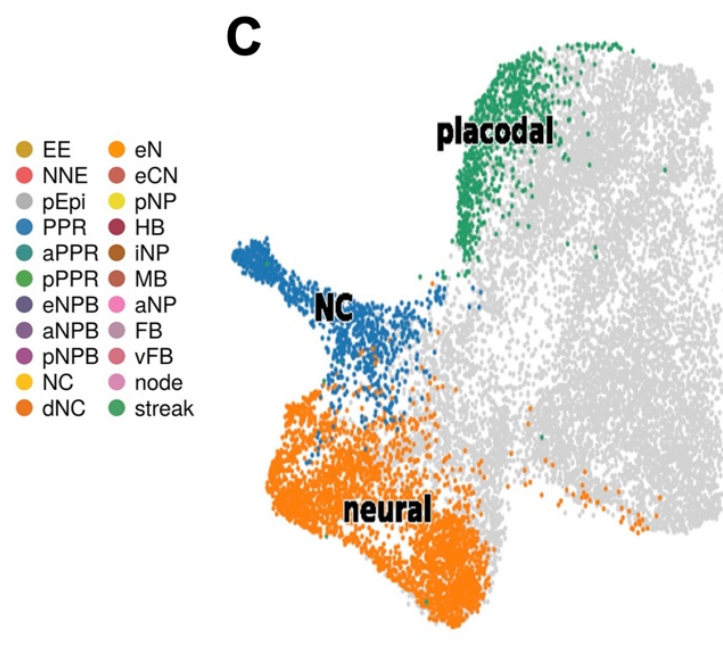

Figure 3-figure supplement 1. (A) Gene modules calculated across the full dataset (full list of gene modules available in Figure 3-Source Data 1). Gene modules were unbiasedly filtered to include those that only display differential expression between cell states and not between sequencing batches and then further filtered to include only those that are differentially expressed between the neural, neural crest and placodal cell states at ss8. 9 gene modules remained after filtering. (B) UMAP plot of the full dataset overlaid with RNA velocity vectors depicting the predicted directionality of transcriptional change. Cells coloured by cell state. (C) Selected terminal states (placodal, neural crest and neural) used for CellRank lineage inference. 


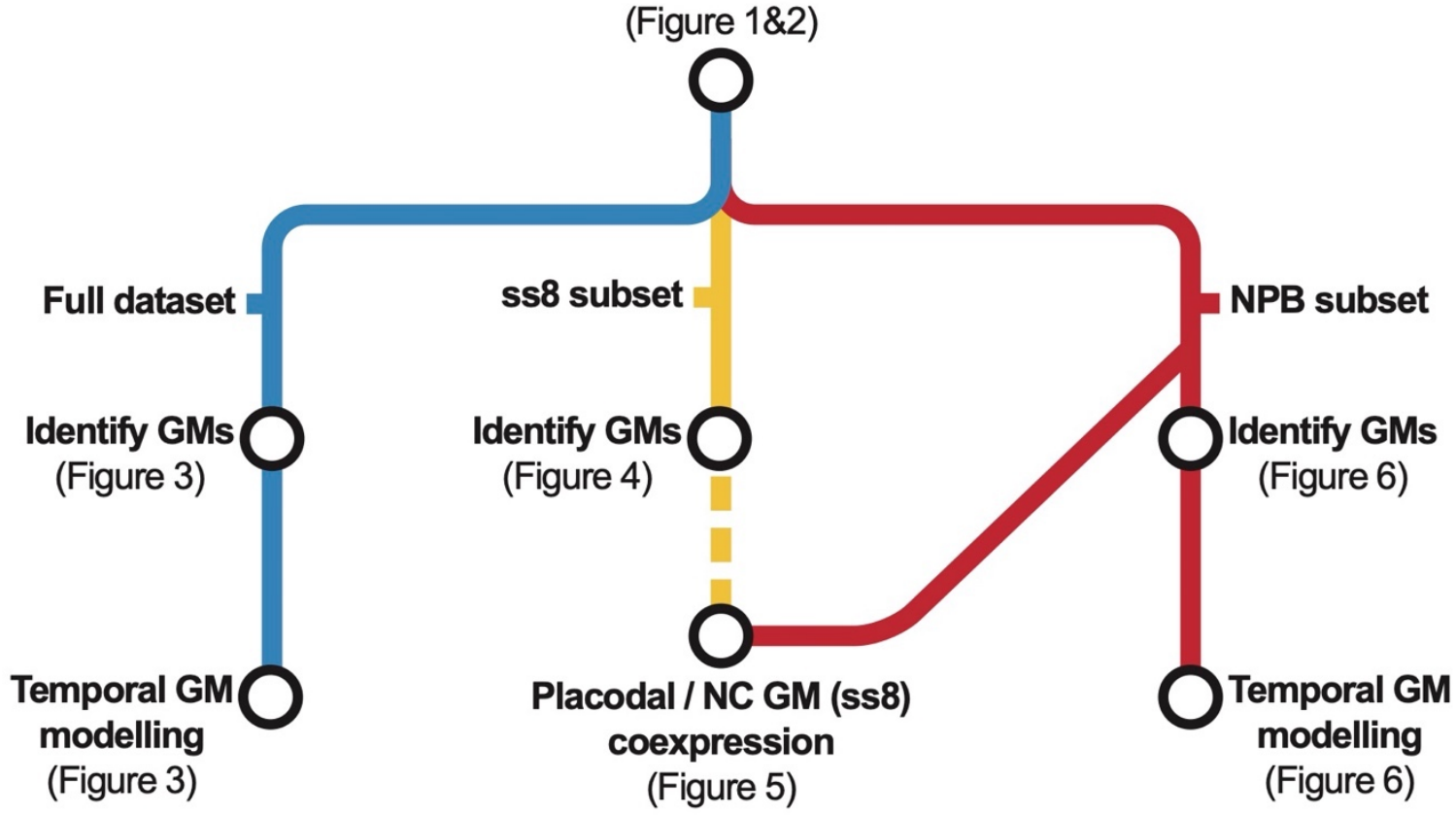

Figure 3-figure supplement 2. (A) Workflow diagram illustrating the different dataset subsets used in each of the main figures. 
A

Bin 1

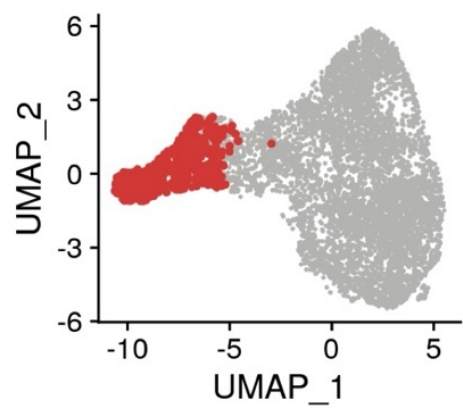

Bin 4

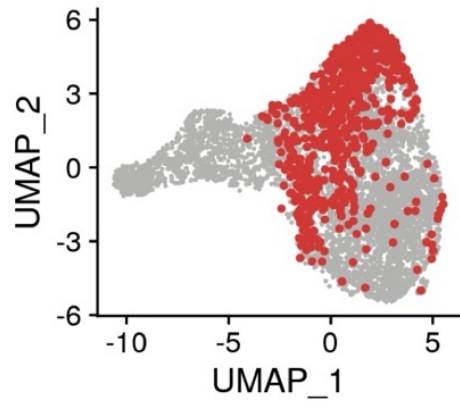

Bin 7

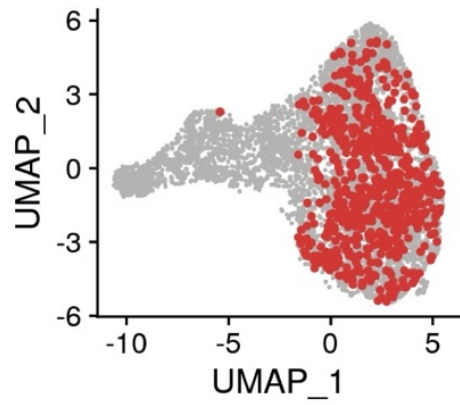

Bin 10

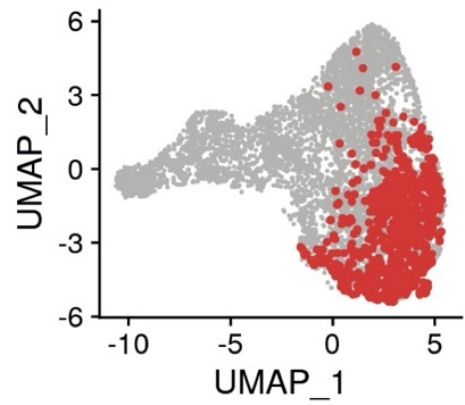

Bin 2

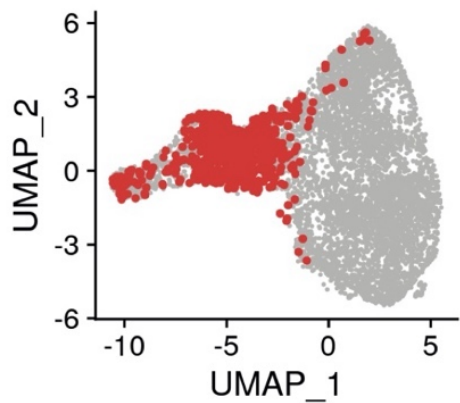

Bin 5

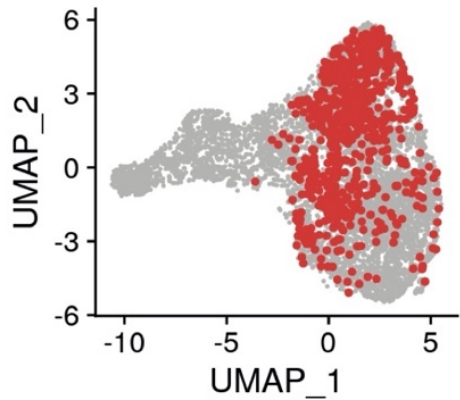

Bin 8

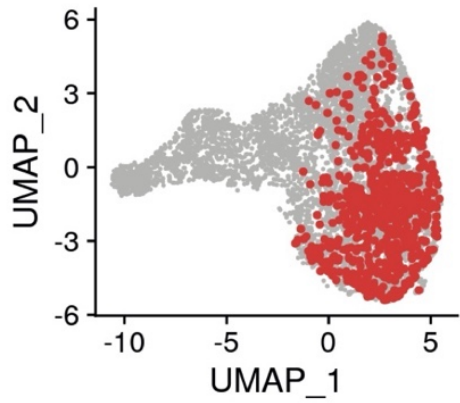

Bin 3

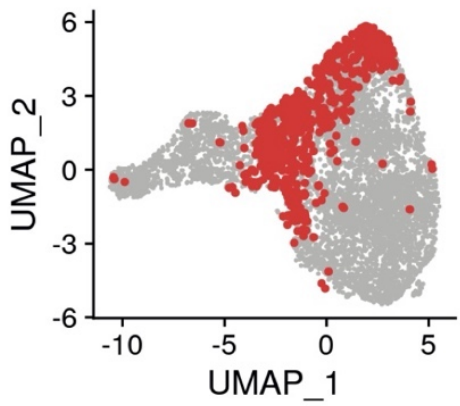

Bin 6

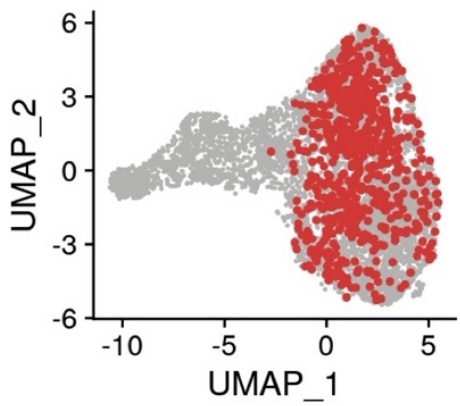

Bin 9

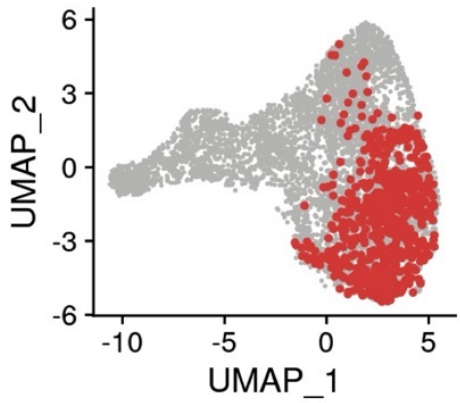

Figure 5-figure supplement 1. (A) UMAPs of the NPB subset. Cells from the NPB subset were ordered by their expression ratio of placodal and neural crest gene modules and then separated into 10 evenly sized bins (Figure 5D). Cells belonging to each of these 10 bins are displayed on the UMAPs. 
A

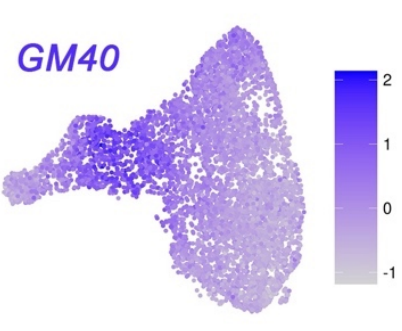

B

$\mathbf{D}_{G M 43}$

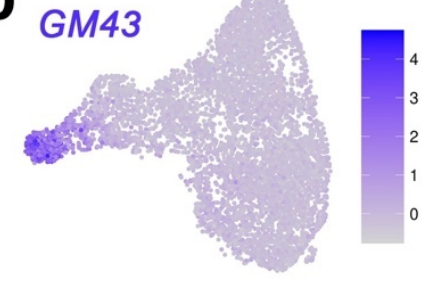

C

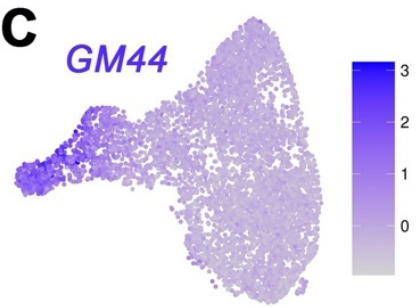

Figure 6-figure supplement 1. (A-E) Feature plots of average gene module expression used for the co-expression analysis in Figure 61.

$\mathbf{E}$

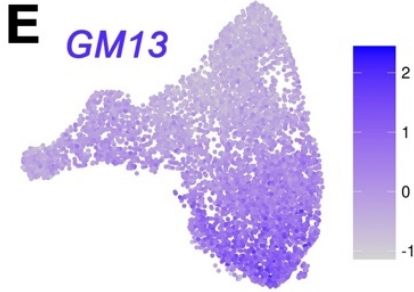



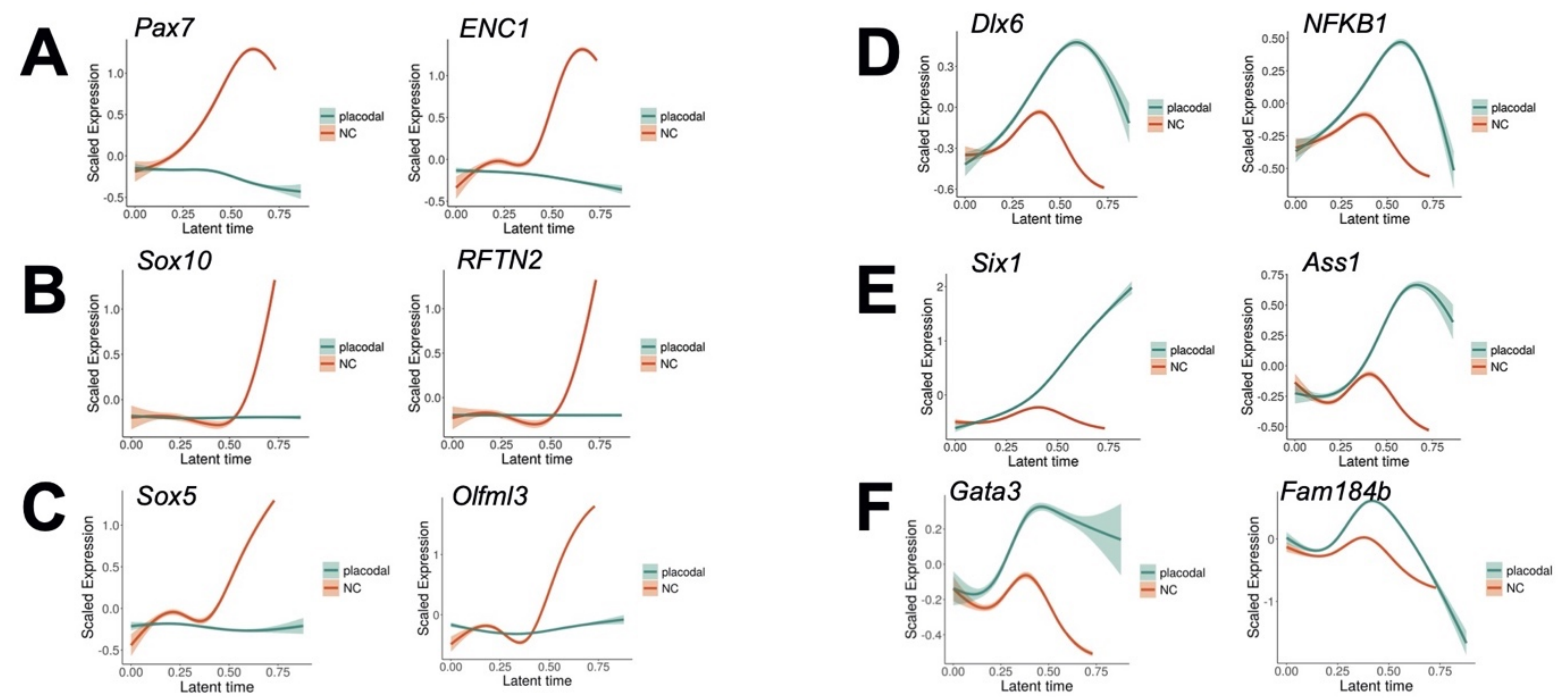

Figure 6-figure supplement 2. (A-F) GAMs are weighted by the absorption probability of each cell towards one of the two fates (placodal or neural crest). Well-characterized genes are shown on the left and novel genes with similar expression dynamics are shown on the right of each panel. 